\title{
Examining Design Choices of Questionnaires in VR User Studies
}

\author{
Dmitry Alexandrovsky ${ }^{*, 1}$, Susanne Putze ${ }^{*, 1}$, Michael Bonfert ${ }^{1}$, Sebastian Höffner ${ }^{1}$, \\ Pitt Michelmann $^{1}$, Dirk Wenig ${ }^{1}$, Rainer Malaka ${ }^{1}$, Jan David Smeddinck ${ }^{2}$ \\ ${ }^{1}$ Digital Media Lab, University of Bremen, Germany \\ ${ }^{2}$ Open Lab, School of Computing, Newcastle University, UK \\ \{dimi,sputze,bonfert,shoeffner,pitt,dwenig,malaka\}@uni-bremen.de Jan.Smeddinck@newcastle.ac.uk
}

\begin{abstract}
Questionnaires are among the most common research tools in virtual reality (VR) user studies. Transitioning from virtuality to reality for giving self-reports on VR experiences can lead to systematic biases. VR allows to embed questionnaires into the virtual environment which may ease participation and avoid biases. To provide a cohesive picture of methods and design choices for questionnaires in VR (INVRQ), we discuss 15 INVRQ studies from the literature and present a survey with 67 VR experts from academia and industry. Based on the outcomes, we conducted two user studies in which we tested different presentation and interaction methods of INVRQS and evaluated the usability and practicality of our design. We observed comparable completion times between INVRQS and questionnaires outside VR (OUTVRQS) with higher enjoyment but lower usability for INVRQs. These findings advocate the application of INVRQS and provide an overview of methods and considerations that lay the groundwork for INVRQ design.
\end{abstract}

\section{Author Keywords}

Virtual reality; VR; user studies; in-VR questionnaires; inVRQs; research methods.

\section{CCS Concepts}

-Human-centered computing $\rightarrow$ Virtual reality; HCI design and evaluation methods; Empirical studies in HCI; User studies;

\section{INTRODUCTION}

The notable rise of a new generation of virtual reality (VR) systems in recent years opened up new methods and interventions for researchers across many different areas. These range from highly immersive stimulus-response studies $[36,60]$ over spatial navigation $[147,175]$ and embodied cognition $[149,154]$

\footnotetext{
*The authors contributed equally.
}

(c) the authors, 2020. This is the author's version of the work. It is posted here for your personal use. Not for redistribution.

The definitive version was published as:

Dmitry Alexandrovsky, Susanne Putze, Michael Bonfert, Sebastian Höffner, Pitt Michelmann, Dirk Wenig, Rainer Malaka, and Jan David Smeddinck. 2020. Examining Design Choices of Questionnaires in VR User Studies. In Proceedings of the 2020 CHI Conference on Human Factors in Computing Systems (CHI '20), 1-21. https://doi.org/10.1145/3313831.3376260 to exposure therapies $[47,56,146]$, exercising [165, 202], education $[18,104]$, work collaboration [7,97], and other forms of social interaction [5,99]. Typically, mid- and post-experience measures are collected via subjective responses on questionnaires [103]. Furthermore, the development and evaluation of VR experiences for entertainment or serious purposes also frequently relies on questionnaires. To fill out conventional paper- or computer-based forms, the subjects need to take off the head-mounted display (HMD) and change the domain from virtual to physical reality [83]. This often leads to temporal disorientation and loss of sense of control [91]. Accordingly, questionnaire results are likely biased to a degree that is difficult to quantify and likely varies from case to case. Such undetermined bias is highly problematic for many types of research and evaluations.

In contrast to the physical domain, alternate reality technologies allow for the embedding of questionnaires directly into the environment. While the transition from VR to answering, for example, paper-based questions presents a drastic change of context, embedding question-items in VR offers an opportunity to stay closer to the context of an ongoing exposure than out-of-VR research setups and avoid a break in presence (BIP) $[83,137]$. Especially for measures where the self-reporting needs to be administered as soon as possible after the treatment and may be especially sensitive to differences in study setups, such as common measures for presence [155], immersion [82] or flow [35, 183], it appears crucial to give careful consideration to the interaction modalities around delivering question-items and gathering responses in order to foster the adequate interpretation of individual research outcomes and for fostering replicability.

Schwind et al. observed a higher consistency of self-reported presence when administering questionnaires in VR. The authors highlight that the effects of using questionnaires in VR are unclear, pointing out that the commonly applied measures were not validated for VR studies [159]. These considerations motivate our investigation on questionnaire practices in contemporary VR user research. We investigate whether authors employ comparable terminology and reflect their choices with regard to questionnaire presentation and response collection mechanisms. To date, VR user research does not have a shared range of common administration procedures, welldefined classification schemes, or standardized toolkits for presenting questionnaires in VR user studies that could guide 
such considerations and make it easier to contrast against other work.

To build an understanding of the current practices and to lay the groundwork for future efforts around developing practical toolkits, we based this work on the following research questions:

RQ1 How are questionnaires and individual question-items applied in contemporary VR user research?

RQ2 What are the considerations around - and possible advantages of - administering questionnaires in VR?

RQ3 What are appropriate design choices for presentation and interaction with in-VR questionnaires?

To approach the research questions, we conducted: (i) a literature review of 123 publications on VR user studies, (ii) a survey with 74 VR experts, (iii) a preliminary design study to compare different presentation and interaction methods, (iv) a user study $(n=38)$ of a revised iteration of our in-VR questionnaire tool, comparing it to a on-screen response system. This aggregated examination of the literature review and expert survey allows us to extract a detailed picture of proceedings of VR user research, compensating for incomplete reporting in publications and for sampling effects [123]. Based on these insights, we iteratively implemented an in-VR questionnaire tool and applied it in 2 user studies to investigate design options and effects of questionnaire administration in VR.

This work focuses on self-reporting methods. Other more objective measures (e.g. behavioural or biosignals) provide more reliable data [79] and are less likely to be affected by BIPs. We also did not take qualitative analyses into account since the procedures differ notably from quantitative studies. By providing a coherent survey of questionnaire methods in VR through a literature analysis, expert interviews, and an empirical investigation of in-VR questionnaire (INVRQ) designs, our research can inform the discourse around questionnaire methods in VR research, structure design considerations on VR user research and also inform the design and implementation of practical questionnaire toolkits that are relevant to both research and industry.

\section{STATE OF THE ART}

Due to its immersive nature and a wide variety in technical setups, VR with HMDs requires careful deliberation by researchers aiming to conduct studies with human subjects. In this section, we review methods and practices for questionnaires in human-subject research followed by a consideration of the VR-specific technicalities around moving between worlds and their effects on question asking.

\section{Questionnaires in Human Subject Research}

Questionnaires are an important source of information for evidence-based research $[12,45,103]$. They embody selfreports and therefore gather the participants' subjective experiences [45]. Question types in surveys can be divided into unstructured and structured questions [144]. Structured questions allow for a clear classification of the responses (see [152] for a more detailed discussion), while unstructured or openended questions allow the subjects to respond freely. This type of question is more exploratory and allows for a broader understanding of phenomena [103], while also requiring more effort from the respondents. Survey methodologies received much attention in the literature and their advantages or drawbacks are widely explored $[13,17,163]$. Reliable (consistency of the measurement) and validated (measuring the right construct) questionnaires are vital for reproducible and consistent research [17]. Choi and Pak [32] list 3 groups of potential biases: design of the question, questionnaire design, and administration. Question design covers the effects of poor wording, such as double-barreled questions, negative phrasing or wording that enforces choices [12,32, 103]. Biases of questionnaire design are due to formatting and length of the surveys [4,32] as well as length and structure of the questions [23]. Contextdependent forgetting $[1,58]$ due to environment change [136] biases the responses. We argue that especially in immersive scenarios, a series of random errors can be minimized through consistent administration of questionnaires. Notably, these considerations on questionnaire design are typically contextualized against paper-based or screen-based questionnaires not considering aspects around BIPs or switching between different realities [159].

\section{Moving Between Virtual and Physical Reality}

When individuals are deeply engaged with an activity or absorbed in a virtual environment (VE), they completely block out the world around them [35]. Brown and Cairns [26] identified 3 levels of immersion in games as a scale of involvement: (i) engagement, the lowest level of immersion, (ii) engrossment, when players become emotionally affected by the VR and (iii) total immersion where players are detached from reality. This phase is also associated with empathy for the characters in the game and transfer of consciousness [150] and is linked to the state of flow [35]. A sudden interruption or transition between realities can invoke negative feelings and affect the emotional state [91]. Accordingly, assessing presence during immersive experiences results in more reliable measurements $[21,49]$. In contrast to immersion, presence is a state of mind, describing the feeling of being part of the VE [82, 201]. Presence relies on involvement and immersion [201]. When "returning" from a state of presence in VEs, a real world task is impaired to the degree of immersion and one's ability to re-engage with the "real world" is decreased $[82,171]$.

Thus, leaving the VE is likely to interrupt the presence perception. Schwind et al. [159] investigated the effect of filling out a questionnaire directly in VR. They replicated their lab in VR and asked participants to fill out presence questionnaires in physical reality and in VR after exposing participants to a VE at varying degrees of realism. Schwind et al. could not find significant differences of presence between the 2 questionnaire modalities. However, the data revealed a lower variance and, thus, higher consistency of the data when the questions were answered in VR. This is in line with evidence from the literature that support the assessment of questions in VR [83,91].

Frommel et al. observed a significant effect on presence when questionnaires are integrated in the game context [53]. Similarly, Shute discussed how to embed questionnaires into games without disturbing the game flow [169]. These considerations 
With Q, not reported: $[2,15,16,20,27,30,31,33,34,38,42,43,50-52$, 54, 63, 67-70, 73-76,86, 89,90,92,93,95, 101, 105-107, 109-111, 115, 117, 120,122, 124-126,130-132, 134, 135,139-143, 145, 151,156,164, 167, 173, 174, 178-181, 184-186, 188, 190, 192, 193, 195, 199, 200, 204]

OUTVRQS: [3, 8, 29, 77, 84, 94, 98, 114, 121, 138, 157, 162, 176, 177, 187, 191, 196, 197]

INVRQS: [6, 28, 37, 44, 55, 66, 85, 100, 108, 127, 148, 158-160, 198]

Without Q: $[9,11,19,22,40,59,71,91,133,153,182,194,205]$

Table 1. Overview of all papers reviewed, organized by whether a questionnaire was used and how it was presented

can guide the choice of adequate levels of embeddedness, and a general decision whether to remain in VR or to exit VR when asking study participants to respond to questionnaires or individual question-items.

\section{LITERATURE REVIEW}

For our literature review we searched in digital libraries [10, 80] that host proceedings of high quality conference series about human-centric methods and VR applications. Namely, we scanned ACM CHI, ACM CHI PLAY, ACM VRST, IEEE VR and IEEE 3DUI for the years 2016-2019. We deliberately chose this timeframe to gain consistent insights on contemporary resarch methods and the release of the HTC Vive in June 2016 [78] substantially changed HMD use in VR user studies. The search included publications until July 2019, considering papers categorized with any of the keywords "virtual reality", "head mounted display", "virtual environment", "user study", and "questionnaire". Only papers with abstracts that mention a VR application with HMDs and explicitly mention some form of evaluation with users or empirical user study were added to the list.

In total we reviewed and evaluated 123 research papers, see Table 1. The papers are organized following whether they use questionnaires for measurement or not. We also categorized the different representations of questionnaires: in-VR questionnaires (INVRQ), out-VR questionnaires (OUTVRQ) and those who did not include a report of the presentation method.

\section{Analysis}

To investigate common procedures of questionnaires in VR user research, we analyzed both the system design and the study design. We paid special attention to the treatment of questionnaires or individual question-items and to the description regarding transitions between VR and physical reality. Further, we looked at the nature of the VR experience. These factors were considered as discriminatory for VR user research from classic, less immersive interventions. Each paper was examined by 2 of the authors. Disagreements were resolved in discussions.

\section{Uses of VR Hardware}

Established desktop VR devices, such as the HTC Vive (63) and Oculus Rift (42), are most commonly used in the papers reviewed followed by mobile device powered HMDs, e.g. Samsung Gear VR (9) and Google Cardboard (4). 3 publications did not report the device used. The input modalities and devices used in the VR applications are mainly native VR controllers (e.g. Oculus Touch, HTC Vive Controller) (49)

\begin{tabular}{llll}
\hline & Interaction & Presentation & Questionnaire (Extent) \\
\hline$[6]$ & Gamepad & n.a. & well-being [44] (SI) \\
{$[28]$} & Gamepad & HUD (Fig. 1b) & well-being [44] (SI) \\
{$[37]$} & Orally & HUD & custom (MI) \\
{$[44]$} & Gamepad & World (Fig. 1e) & well-being (SI) \\
{$[55]$} & n.a & n.a. & presence rating [21] (SI) \\
{$[66]$} & VR controller & World & well-being [44] (SI) \\
{$[85]$} & VR controller & HUD (Fig. 1a) & custom (SI) \\
{$[100]$} & VR controller & World & custom (SI) \\
{$[108]$} & Full body & n.a. & IAT [64] (MI) \\
{$[127]$} & VR controller & World (Fig. 1d) & NASA-TLX [72] (MI) \\
{$[148]$} & Freehand & n.a. & custom (MI) \\
{$[158]$} & Freehand & World (Fig. 1c) & PQ [201] (MI) \\
{$[159]$} & VR controller & World (Fig. 1f) & SUS [189], IPQ [155], \\
& & & PQ [201] (MI) \\
{$[160]$} & Freehand & World (Fig. 1c) & PQ [201] (MI) \\
{$[198]$} & Freehand & Body (Fig. 1g) & PANAS [96] (MI) \\
\hline
\end{tabular}

Table 2. Examples of INVRQs with their realization (interaction and presentation), the questionnaire used and its extent (multi-items (MI) vs. single-item (SI)), if reported in the publication (n.a. otherwise)

followed by freehand interaction (e.g. Leap Motion or Microsoft Kinect) (35) and general purpose input devices (e.g. game controller, keyboard, mouse, stylus, smartwatch, and touch screen) (25).

\section{Questionnaire Assessment}

110 out of 123 papers report having used questionnaires in their VR user studies. Since the use of VR devices entails design decisions regarding the presentation of questionnaires and individual question-items, we surveyed the documentation of such decisions in the respective papers. 77/110 do not report how they presented the questionnaires to their users. 13 papers report that the participants filled out the questionnaire after leaving the VE but do not describe whether they used paperor screen-based questionnaires. 15 papers report on the usage of INVRQS - either for the whole question asking procedures in the user-study (3) or in combination with OUTVRQS (12).

\section{Cases of In-VR Questionnaires}

15 papers report the use of INVRQs. Some describe the design in more detail. Figure 1 depicts 7 different realizations of INVRQS. Kang et al. (Fig. 1a) used a 2D heads-up display (HUD) overlay with a single question about the user's motion perception between multiple trials in their VE [85]. The user interface (UI) shows a single question with a multi-line question text and 3 buttons for answering choices. As input device, they used a native VR controller [85]. Schwind et al. (Fig. 1c) included the full 32-item PQ [201]. The participants stayed in the VE for the whole duration of the study: on average $58.6 \mathrm{~min}$ [160] and $75 \mathrm{~min}$ [158]. The authors designed a 3D floating UI which appears in front of the subjects showing a one-line text instruction and 4 items on 7-point Likert-scales. Users select answers and navigate the questionnaire with freehand gestures using a Leap Motion [158, 160,161]. In another study, Schwind et al. (Fig. 1f) placed single questions on presence on a virtual PC in the VE, with which a user interacts with the trackpad of a VR controller [159]. Oberdörfer et al. (Fig. 1d) presented the NASA-TLX [72] using a virtual world-referenced representation of the paper-based version. The users interacted using a VR controller with pointing [127]. 


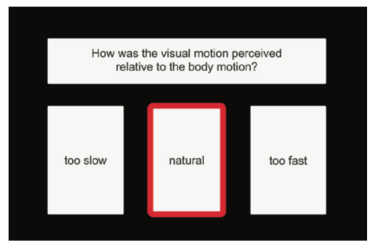

(a) Kang et al. [85]

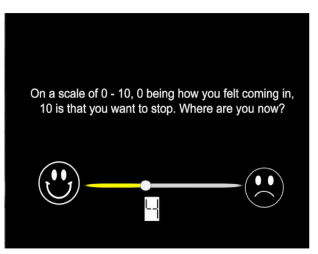

(b) Cao et al. [28]

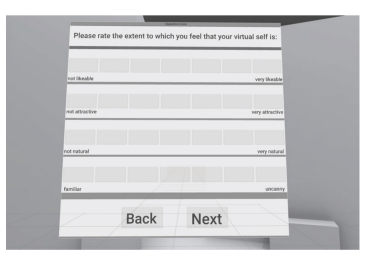

(c) Schwind et al. [161]

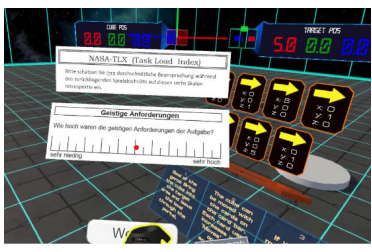

(d) Oberdörfer et al. [127]

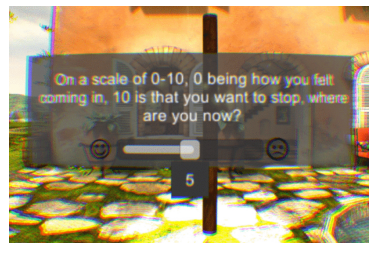

(e) Fernandes and Feiner [44]

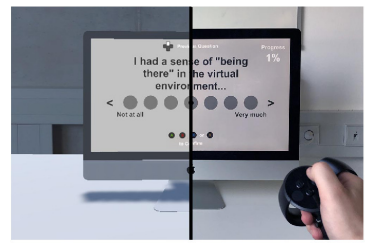

(f) Schwind et al. [159]

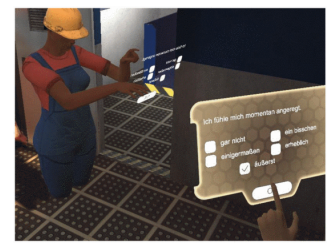

(g) Wienrich et al. [198]

Figure 1. Examples of different realizations of INVRQ: (a) and (b) present the questionnaire using a HUD, (c)-(f) use a world-referenced questionnaire, and (g) presents the questionnaire attached to the body.

Wienrich et al. (Fig. 1g) presented a body-referenced INVRQ [198]. The questionnaire was displayed on a 2D floating UI with a 20-items PANAS [96] attached to the hand of the virtual character. They combined the in-experience measurement with further out-VR measurements and oral answering of the Fast Motion Sickness Scale (FMS) [88]. Fernandes and Feiner (Fig. 1e) is the earliest example of INVRQs in our sample. The authors applied a 10-point Likert-scale slider on well-being where subjects could stop the experiment by selecting the maximum value of discomfort [44]. [28] (Fig. 1b), [6] and [66] adapted this method in their works in different realizations.

All INVRQ designs differ in their presentation (HUD, worldreference, body-referenced), their extent (single-question vs. multi-item questionnaire), question-item presentation (textbased vs. scales) and interaction modality (pointing, free-hand, trackpad). Table 2 summarizes the variation of the INVRQ designs. The applications of INVRQS cover questions about the subject's well-being, their sense of presence and taskspecific questionnaires, e.g. about task workload or affect.

\section{Discussion}

Our literature analysis shows that comprehensive reporting of questionnaire usage in VR research is frequently neglected. Only Schwind et al. [159] compare INVRQS and OUTVRQS and discuss their effects. This indicates that the field may benefit from building awareness and providing guidelines. We identified 15/123 cases of INVRQS applied in VR user studies. The realizations differ substantially in their presentations and interaction methods. The majority of cases used presentations that contextualized the questionnaires in the VE, either attached to the user, or anchored in world space in a stationary manner. The participants used predominantly native controllers to interact in VR, directly followed by free-hand interaction. In order to better contextualize these literature findings and to collect details about the design of INVRQS and their potential shortcomings, we discuss a further investigation through an online expert survey in the following section.

\section{EXPERT SURVEY}

To augment the insights gained from the literature review, we conducted an expert online survey that evaluates general proceedings of VR user studies and attitudes towards INVRQS. With this additional analysis of the state of the art, we aimed to capture an impression of the actual procedures employed by the researchers independent from possible biases present in publications with space limitations.

\section{Survey Dissemination and Pre-Processing}

We developed a custom survey to capture the general reporting of proceedings of VR user studies as well as experiences and attitudes of the study designers regarding INVRQS. The complete survey is provided in an OSF project ${ }^{1}$. Following informed-consent, it consisted of 22 questions grouped into 5 categories (demographics, general research practice, VR research practice, INVRQ experience and OUTVRQ experience). The survey was designed and distributed using Google Forms. For recruiting expert participants, we extracted a list of authors from the papers (2016-2018) we analyzed in our literature review and sent them personal invitations. We also advertised the survey via social media channels. Over 6 weeks in July and August 2018, we collected 74 replies.

Since the online survey focused on researchers and VR experts, we excluded 4 participants who were not directly involved in VR user studies in the last 24 months and 3 participants who indicated that they do not generally use questionnaires as measures in their VR user studies. Moreover, we corrected obvious spelling mistakes to facilitate accurate counts of established terms (e.g. hardware or questionnaire names). For anonymized analysis, we removed time stamps and added unique identifiers (E1-E74). The reported analysis focuses only on questions that are most relevant to our research questions.

\section{Analysis}

After data cleaning, the analyzed data set consisted of 67 full set responses. Based on participant indication, we sampled

\footnotetext{
1 https://osf.io/f5qy7/
} 
responses from 13 different countries of residence (Q3). With 20 participants from Germany, 9 responses each from the USA and Portugal and 4 responses each from Denmark and France, the majority of our sample came from northern-hemisphere countries. As a result of the sampling strategy, the expert sample stems predominantly from academia. The participants indicated to be holding the following positions (Q2): $22 \mathrm{Ph} . \mathrm{D}$. students, 16 professors (full, associate, assistant or equivalent), 10 undergraduate students, and 8 Postdocs. The remaining 11 participants provided individual answers, such as research engineer or research fellow. On average, the participants indicated to be rather experienced with designing user studies (Q4). On a 6-point Likert-scale (0 to 5): $M=3.76, S D=1.18$. The majority (56) were directly involved with conducting $1-5$ VR user studies in the last 24 month while 8 experts indicated involvement with between 6 and 10 studies. Only 3 indicated more than 10 VR user studies (Q9). Our sample consists of a diverse spectrum within the group of academic researchers who have a sound background on VR user research allowing us to interpret the responses as an expert evaluation.

\section{$V R$ research}

The experts' most commonly used VR devices (Q10, multiple choice) in the last 24 months were desktop VR devices (HTC Vive (55) and Oculus Rift (37)) or mobile VR devices (Samsung Gear VR (15) and Google Cardboard (11)). Other devices were mentioned occasionally. Only 4 participants used neither HTC Vive nor Oculus Rift. The most commonly used input modalities (Q11, multiple choice) reported for the general interaction with VR are mainly native VR controllers (50) and freehand interaction (e.g. Leap motion or Microsoft Kinect) (31) followed by gaze interaction (19) and general purpose input devices (e.g. game controller, keyboard, mouse, stylus or smartwatch) (34). 6 participants reported using custom controllers that were not further defined.

\section{Questionnaires}

We asked our participants how they usually present questionnaires in their user studies. 29 use a separate screen outside the VR, 21 paper questionnaires, 6 embed the questionnaire in VR, 2 use oral answers and 9 use mixed methods (Q13). Validated questionnaires (OUTVRQS) are very common in VR user research (Q12). Independent of the form of presentation, the experts reported they encounter some difficulties with questionnaires in VR user studies (Q16): 14 experts reported problems with the questionnaires in general, such as ambiguous question items in validated questionnaires, e.g. PQ, or the length of questionnaires, e.g. SSQ [87], NASA-TLX [72], especially for use in measurements between trials. 7 experts reported problems of their participants when moving from VR to reality for answering the questionnaires, namely the lack of immersion (4) and the temporal effort (3).

29/67 experts have tried in-VR questionnaires (INVRQ users). Most of them (25) tried fully interactive questionnaires that are both presented and answered in VR (Q18, single choice). All experts rated the usefulness of INVRQS (Q17) on a 6point Likert scale $(0-5)$. They rated the usefulness with $M=2.97, S D=1.58$ which is significantly higher than the midpoint 2.5 (one-sided independent sample t-test $t_{65}=2.41$,

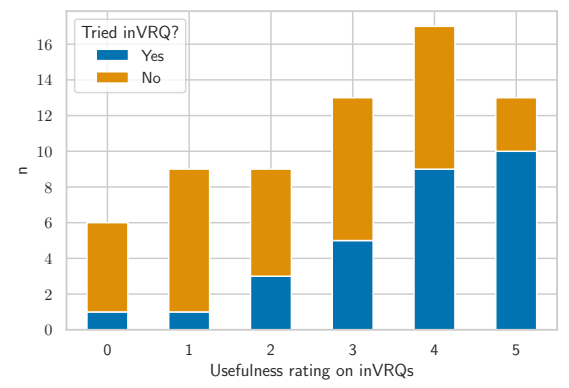

Figure 2. Ratings of the usefulness of INVRQS (Q17, scale: 0 to 5, 5 being highest) separated by INVRQ experience (Yes/No).

$p<.01)$. For further analysis, the experts were split into 2 sub-groups according to whether they have tried an INVRQ in their research setups (Q18) or not $\left(M_{\text {Yes }}=3.72, S D_{\text {Yes }}=1.33\right.$ and $\left.M_{N o}=2.39, S D_{N o}=1.55\right)$, cf. Figure 2. A two-sided independent sample t-test showed a significant difference between prior INVRQ experience and the usefulness rating $\left(t_{65}=3.69, p<.01\right.$, Cohen's $\left.d=.45\right)$. To appropriately weight the responses we further split the experts according to their scientific seniority. Group A: post-docs and professors, Group B: all other participants (presumably more junior researchers). The experts in group A tend to rate the usefulness higher than the other participants: With $M_{A}=3.46, S D_{A}=1.74$ and $\left.M_{B}=2.70, S D_{B}=1.46\right)$. But the difference was not significant $t_{65}=1.91, p=.06$.

\section{In-VR Questionnaires}

14/29 INVRQ users presented the questions in world space as floating displays and 4 used a 2D UI (probably replacing the virtual world) (Q19). 4 experts responded that they presented only 1 question at a time. 14 experts did not observe any difficulties of their participants using INVRQS (Q20). Usability and the "amount of time for filling out the questionnaires" (E44) were major challenges experts came across $(4 \times$ each). Further, "completing long questionnaires can be (...) annoying" (E18) and participants are "impatient (...) and hence made mistakes in the scaled questions" (E5).

\section{Out-VR Questionnaires}

Using Q18 as a branching question, 38 oUTVRQ users were asked to provide reasons for not embedding the questionnaires into the VE (Q21). 19 from that group reported that they do not suspect to benefit from INVRQs. 9 experts reported technical difficulties and 3 exceeding time effort in setting up the embedding for INVRQs. "Time effort to embed into VR much higher than doing the same on paper and harder to change/make additions" (E48). 6 experts mentioned usability problems and 1 sees problems in all these categories. The experts explained their critical opinions of INVRQs further (Q22): They argue, it would reduce the "willingness to answer the questions" (E31) because interaction with the UI in VR is more frustrating and takes longer than on desktop. Especially text entry is seen as a major issue. The experts reason that this might bias the results "towards the choices that are easiest to make through the interface" (E31). Additionally, they mentioned the time effort for implementation and usage: 
"My last study took 45 minutes including questionnaires. It gets very hot and inconvenient under a Vive for such a long time - especially if it's your first time" (E71).

\section{Discussion}

The responses to the survey indicate that experts generally appear to have a positive attitude towards INVRQS. Both our literature review and expert survey demonstrate that there is no common standard for using questionnaires in VR user studies. While the reporting of the presentation type of the questionnaires in the literature is mostly imprecise, our expert survey did not surface a clear standard method for presentation. Thus, regarding RQ1, the state of the art for delivering questionnaires in VR user studies is quite heterogeneous. Also, we found inconsistencies between the literature analysis and the online survey: While only $15 / 123$ papers reported on the usage of questionnaires embedded in VR, 29/67 experts reported that they have tried INVRQs before. Possible reasons for the contrast between the positive attitude of the INVRQ user group and the rare reporting of INVRQS are not intuitively clear. The difference could indicate that a shift may be taking place in the community with many applications of INVRQS not having been published yet. There is, however, the possibility of increased variance due to publication bias that warrants control in future work. Alternatively, the authors of the analyzed corpus may have considered the presentation type to be irrelevant. In contrast to these outcomes, there are experts who have a strong opinion against embedding questionnaires and argue that INVRQS could bias responses. In response to RQ2, the broad range of reasons provided for or against using INVRQS indicate technical challenges, implementation effort, and fear of biases and participant overload. In the following section we investigate these objections from the users' viewpoint and design and evaluate an INVRQ tool.

\section{DESIGN STUDY}

Although many guidelines from "traditional" HCI, cf. [41, 168], can be applied to VR, the third dimension brings its own challenges for UI designers. LaViola et al. [102] point out that there are no standards for 3D UIs yet, such as the ISO 9241110 [81] standards for 2D GUIs. The results from the literature review and from the expert surveys are heterogeneous and do not allow for a definite answer of the usefulness and realization of INVRQS. With regard to RQ3, the goal of this design study was to identify the most usable interface design.

\section{In-VR Questionnaire Design}

Based on the results of our expert survey and general guidelines on traditional UIs [41, 168] as well as UIs in VR [40,62,128,203], we implemented 4 variants of INVRQS. The interfaces differ in 2 dimensions: anchoring (world-referenced and body-referenced) and interaction modality (pointer and trackpad). The world-referenced UI is anchored in the VE and users can freely move around the interface. A body-referenced $\mathrm{UI}$ is attached to a hand-held controller.

To enhance readability, we applied guidelines from research and industry which recommend signed distance field fonts [61, 128]. In line with Dingler et al. [40], we used light glyphs on a dark background. Oculus best practices recommend to avoid HUDs (cf. Fig. 1a, 1b) in favor of UI elements that are settled in the VE, as it overcomes the binocular disparity and allows to contextualize the UI in VR $[102,128]$. This is in line with the statements from the experts who applied INVRQs, as the majority $(32 / 67)$ implemented questionnaires in the world space (see Fig. 1c-1f). In contrast to world-referenced UIs, body-referenced interfaces, as in Fig. 1g, take advantage of the user's proprioceptive sense and can significantly enhance the interaction with the UI [102, 118].

The interaction with the UI varies between a laser pointer - as Oberdörfer et al. [127] applied for their INVRQS - and a clicking interaction where the trackpad of the controller is used to navigate through the questionnaires, similar to Schwind et al. [159]). Oculus guidelines [128] suggest a laser pointer with a visible ray-cast and a cursor projected on the UI as an appropriate and intuitive method to interact with UI in VR. This is reflected in general guidelines which suggest better performance in terms of speed, accuracy and cognitive demand [112, 113, 119]. In contrast, navigating a UI using a trackpad promises to be more efficient when the UI is close to the users since they are not required to twist their arms for aiming.

The 4 designs that emerge from the 2 dimensions anchoring (world, body) and interaction (pointer, trackpad) cover a wide range of designs that are applicable to INVRQS. We developed all 4 designs iteratively following the same usability guidelines and paying particularly attention for comparability. Our interface supports continuous values (slider), checklists, radio lists, drop downs; and switches. The prototype is implemented in Unity3D with OpenVR. Their interaction and design is demonstrated in the accompanying video figure.

\section{Study Design, Procedure and Tasks}

The study has a $2 \times 2$ within-subject design where users filled out INVRQS using the 4 different versions of the interface: world-pointer (WP), world-trackpad (WT), body-pointer (BP) and body-trackpad (BT). The order of the condition was counterbalanced using Latin Square. First, the subjects were welcomed and informed about the study. The experimenter fit the HMD (HTC Vive) and explained the interaction in the current condition with the native controllers. To provide a context to the VE, we used a sci-fi scene.

To investigate the usability of all response types, we developed a questionnaire which asks for common knowledge facts to ask subjects easy-to-answer but objective questions to calculate correctness. Each condition consisted of a questionnaire that comprised all 5 question types once. Each participant answered 20 questions in total with each question type once per condition. After each condition in VR, the participants took off the HMD and filled out a paper-based System Usability Scale (SUS) [25]. When the participants finished all 4 conditions, the experimenter asked them to put the 4 interfaces into a ranked order and conducted an interview.

\section{Participants}

10 male (age $M=29.9, S D=2.9$ ) subjects from a game jam at the campus participated in the study. For their experience in VR and as VR developer or researcher, the participants gave 
a score on a Likert scale from 1 (no experience) to 5 (high experience). All participants had prior experience in VR and game development $(M=3.6, S D=.84)$. 6 participants developed VR applications or conducted studies in VR $(M=2.3$, $S D=1.33$ ). Although this sample lacks representative diversity, it allows for a deep discussion of the design space while avoiding general issues that could have come up with novices.

\section{Results and Discussion}

For the quantitative metrics (SUS [25] and duration), we conducted a repeated measures ANOVA with condition as factor. The descriptive statistics and the results of the analysis are in Table 3. Bonferroni corrected pairwise comparisons of the SUS [25] scores revealed significant differences between BP and BT $(p<.01)$, between BP and WP $(p<.01)$, and between WP and WT $(p<.01)$. For the required time, Bonferroni corrected pairwise comparisons revealed significant differences between BP and BT $(p<.01)$, BT and WP $(p<.01)$, and between WP and WT $(p<.05)$. On the subjective rankings, the participants rated the world-pointer setup significantly higher $\left(F_{3,36}=13.27, p<.001\right)$.

$6 / 10$ subjects stated that the laser pointer is easy and intuitive to use. In contrast, the majority perceived the trackpad as tedious and confusing. 3/10 participants liked being able to move the questionnaire. But $4 / 10$ participants reported they were confused by the body-referenced interface and stated the movement as unpleasant. The world-anchored UI was stated as less demanding than the body-referenced $(3 / 10)$.

Completion time, SUS scores [25] and the interviews clearly show the world-referenced anchoring with the laser pointer interaction is easiest to use and therefore the best candidate to investigate the concerns raised by the experts.

\section{USER STUDY}

To assess whether usability and duration concerns about INVRQS raised by the experts hold true and to provide guidance on question-asking methods in VR, we conducted a user study in which the participants shot balloons with bow and arrow in a VR archery game and then filled out INVRQs as well as questionnaires presented on a notebook (OUTVRQ). The aim of this study was to evaluate adequate design choices of INVRQS we identified in the design study and to capture the users' perspectives on INVRQs. In contrast to previous work by Schwind et al. [159], our user study applies the questionnaires in a realistic study setting without replicating the lab space virtually.

\section{The Questionnaire Tools}

We implemented the INVRQ with world-based anchoring and laser pointer interaction (Fig. 3). We refined the overall readability, i.e. font size, contrast, spacing and positioning

\begin{tabular}{lrrrrr}
\hline & WP ( M, SD) & WT (M, SD) & BP (M, SD) & BT (M, SD) & F(3,27) \\
\hline SUS [25] & $91.25,8.99$ & $64.25,24.09$ & $79.00,14.49$ & $52.25,19.52$ & $11.15^{* *}$ \\
$\mathrm{t}$ (min) & $0.61,0.12$ & $1.00,0.27$ & $0.69,0.33$ & $1.19,0.23$ & $14.92^{* *}$ \\
\hline
\end{tabular}

Table 3. Descriptive statistics and RM-ANOVA of the design study for the 4 conditions world-pointer (WP), world-trackpad (WT), bodypointer (BP) and body-trackpad (BT) on SUS [25] and time. ${ }^{* *}$ p<.01.

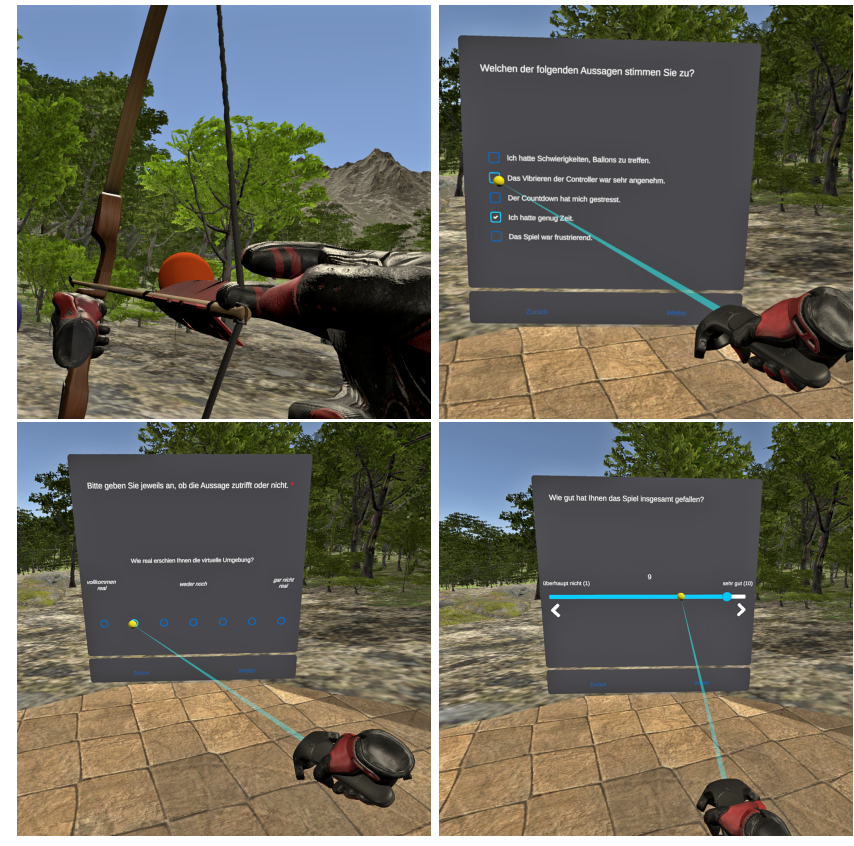

Figure 3. Screenshots of the archery task. The world space-anchored INVRQ is filled out using the HTC Vive controller as a laser pointer.

of the UI in the VE. The tool supports sliders, radio lists, radio grids and check lists. We omitted drop downs and freetext fields since these elements rarely appear in standardized questionnaires, as the literature review shows. If needed, drop downs can be represented using a radio list, and free-text input can be approximated with voice recording.

The OUTVRQs were realized using the questionnaire tool LimeSurvey [57]. It was presented on a 15" notebook with external keyboard and mouse with default speed.

\section{Measurements}

After the archery game, we measured presence in the VE using IPQ [155] on a Likert scale with the subscales general presence (GP), spatial presence $(S P)$, involvement $(I N V)$ and experienced realism (REAL). Furthermore, we asked the participants to rate the game and the perceived control over the bow on a 10-ticks slider. For demonstrating a greater variety of question types, we additionally included questions about the VE ( $1 x$ numerical, $4 x$ single choice with $2-5$ items, $4 x$ multiple choice with 5 and 16 items).

To evaluate the workload and the usability of the INVRQ tool, we employed the raw NASA-TLX [72], using a 20-ticks slider to measure physical, cognitive and temporal demand, as well as performance, effort, and frustration. Usability was measured with the UMUX [46], a four-item questionnaire providing comparability with the SUS [25] $(r=.96)$. As an objective performance metric, we logged the exact time. To get detailed insights from the users' perspective, we conducted a semi-structured interview at the end of a session.

\section{Study Design and Procedure}

The study compares the usage of INVRQs to digital OUTVRQS. To provide a realistic study setting, we designed a 

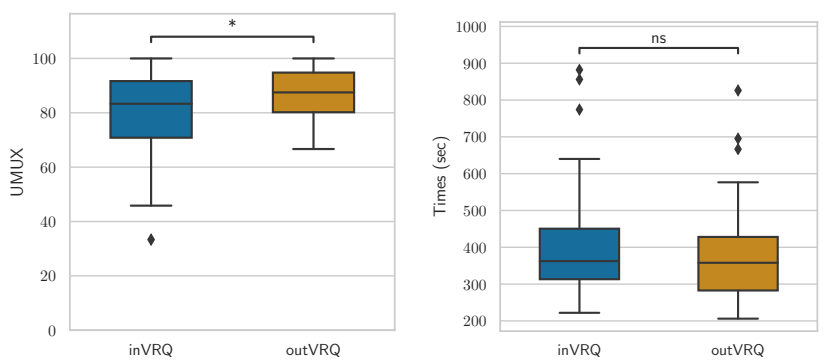

Figure 4. Ratings of usability on UMUX [155] (left) and of completion times (right) for both conditions.

balloon archery game as an immersive VR experience (see Fig. 3). We chose this task because (i) it is engaging and requires the participants to focus, promising an immersive VR experience, (ii) the interaction is easy to learn but differs from the pointing interaction used for answering the INVRQs; thus, the task is less likely to produce carry-over learning effects. For the archery task, we used free Unity3D assets and implementations from the SteamVR Interaction System. The VE consists of a round platform with 3 pillars that display instructions that guide through the experiment. The platform is surrounded by 12 spawn points for the balloon targets and it is situated in a realistic environment with mountains, trees, a river and high resolution textures. The questionnaire interaction builds on the laser pointer by SteamVR and the GUI interaction by HTC Vive.

The study followed a within-subject design with the conditions INVRQ and OUTVRQ in randomized order. After the participants were informed about the procedure, they signed a consent form. The experimenter fitted the HTC Vive Pro HMD. Then the participants played a tutorial round in which they had to hit 5 balloons followed by a 90 s round where they should hit as many balloons as possible. After the archery task, the participants filled out questionnaires using the corresponding tool for their first condition (INVRQ, OUTVRQ). Afterwards, the participants repeated the game and questionnaires using the other questionnaire method. We encouraged them to take a 2 min break in between. After both conditions, the experimenter conducted a semi-structured interview followed by a paper-based demographics questionnaire. Finally, the participants were orally debriefed. The study, including game, questionnaires and interview, was conducted in German and took around $45 \mathrm{~min}(\approx 11 \mathrm{~min}$ in $\mathrm{VR})$.

\section{Participants}

We advertised the study on campus, social media and in lectures and conducted it in July 2019. In total, 38 participants (age: $M=27, S D=10.8 ; 16 \mathrm{f}, 22 \mathrm{~m}, 0$ other; 20 started with the INVRQ condition) volunteered for our study. Most participants were students. 21 participants used vision aids in VR, 1 participant has a light dyschromatopsia. The sample has a broad range of prior VR experience: 6 participants use VR regularly, 27 occasionally and 5 never used VR before. 19/38 participated in other VR user studies previously and 3 participants used INVRQS before. We detected no outliers regarding demographics and task completion times.

\section{Results}

Performance, Presence and Rating of the VR Experience

On average, participants shot 24.71 balloons $(S D=10.33)$ in the first round and improved by $6.34(S D=6.01)$ balloons in the second round. We obtained presence on the IPQ [155] on a 7-point Likert scale (0-6). To determine if the measures deviate from neutral, we performed a two-sided one-sample t-tests against the midpoint 3 . The results show a positive difference for GP $\left(t_{37}=14.93, p<.001\right), \mathrm{SP}\left(t_{37}=22.53, p<.001\right)$ and INV $\left(t_{37}=5.99, p<.001\right)$, but no difference for REAL $\left(t_{37}=-48, p=.63\right)$. There was no significant effect of condition on any IPQ subscales (see Fig. 5a). Like Schwind et al. [159], we performed a t-test to compare the variances of both conditions on all IPQ subscales; the differences were not significant $\left(G P_{v a r}: t_{37}=-.52, p=.60, S P_{v a r}: t_{37}=-.05, p=.96\right.$, $\left.I N V_{\text {var }}: t_{37}=1.47, p=.15 R E A L_{v a r}: t_{37}=.17, p=.87\right)$.

Further, the participants rated the interaction with the bow $(M=7.89, S D=1.70)$ and how they liked the game $(M=8.0$, $S D=1.73$ ) on a 10 -ticks analog scale from 1 (not at all) to 10 (very much). A one-sample t-test revealed significant differences against the midpoint (5.5) for bow control $\left(t_{37}=15.01\right.$, $p<.001)$ and game ratings $\left(t_{37}=14.86, p<.001\right)$, but no effect of conditions neither for bow control nor game rating.

\section{Duration and Self-reports on Usage of the Questionnaires}

Figun 4 shows plots of UMUX scores and answering time. On average, participants required $6.77 \mathrm{~min}(S D=2.69)$ to fill out the questionnaires in VR and $6.30 \mathrm{~min}(S D=2.26)$ on the notebook (without the time for taking off the HMD). There was no significant difference of condition on duration of filling out the questionnaires $\left(t_{37}=-1.05, p=.29\right)$. Nevertheless, in the post-experiment interviews, 5 participants perceived filling out the questionnaires in VR as faster. 2 had the impression the questionnaires in VR would be shorter. The IPQ completion times did not differ significantly (INVRQ IPQ: $M=146.39 \mathrm{~s}, S D=65.46$ ) in comparison to the ones reported by Schwind et al. (VR IPQ: $M=146.94 \mathrm{~s}, S D=63.2$ ) [159]: $t_{37}=.04, p=.97$. On the UMUX, the participants rated the questionnaire tools' usability. Both systems were rated positively (INVRQ: $M=77.35, S D=18.35$, OUTVRQ: $M=86.21$, $S D=9.4)$. The difference between the conditions was significant $\left(t_{37}=2.82, p<.01\right)$. In accordance with Grier [65], the participants experienced low to medium workload on the NASA-TLX [72] (INVRQ: $M=18.64, S D=11.38$, OUTVRQ: $M=14.40, S D=9.42)$. Paired t-tests revealed significant differences on physical demand $\left(t_{37}=4.14, p<.01\right)$ and effort $\left(t_{37}=3.00, p<.01\right)$ subscales but not on mental demand $\left(t_{37}=1.42, p>.05\right)$, temporal demand $\left(t_{37}=1.15, p>.05\right)$, performance $\left(t_{37}=1.48, p>.05\right)$ or frustration $\left(t_{37}=1.76, p>.05\right)$. The results are depicted in Figure $5 \mathrm{~b}$. We contrasted the mean TLX-scores against Schwind et al.'s values (VR: $M=33.16$, $S D=20.96$, PC: $M=37.77, S D=19.26$ ) [159]. Our data show a significantly lower workload for both corresponding comparisons INVRQ $\left(t_{72}=3.50, p<.01\right)$ and OUTVRQ $\left(t_{72}=6.57\right.$, $p<.01)$ conditions.

\section{Qualitative Results}

We collected relevant statements from the interviews and dynamically generated categories emerging from the material. 


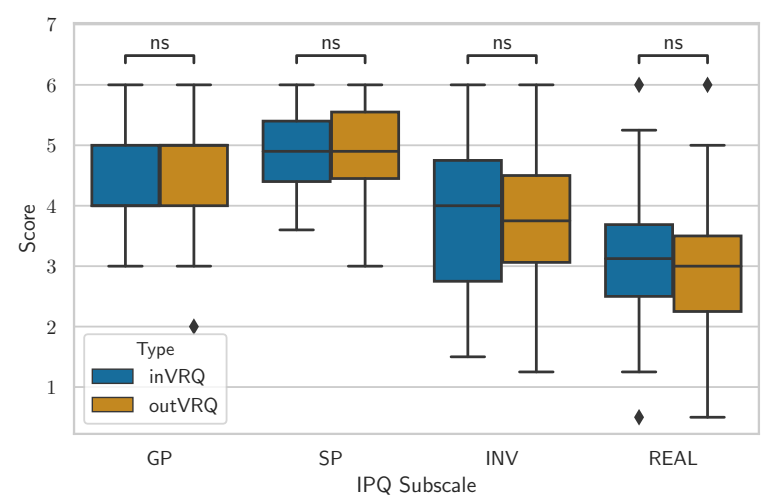

(a) Ratings of presence on IPQ [155] subscales general presence for (GP), spacial presence (SP), involvement (INV) and realism (REAL) split by INVRQS and OUTVRQs conditions.

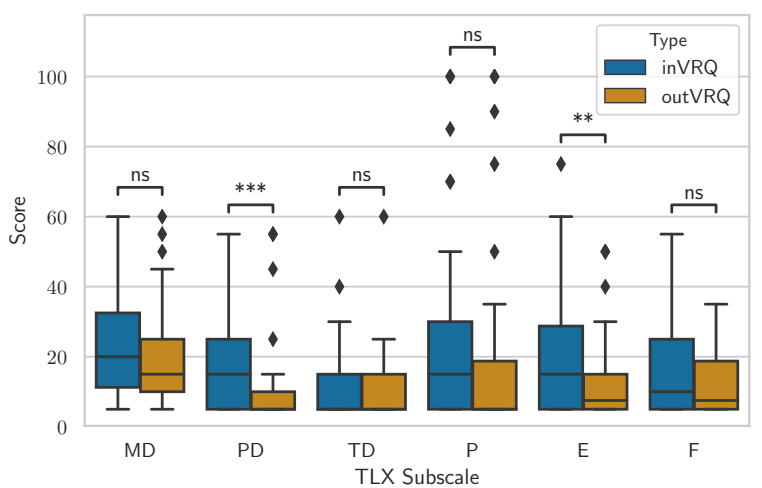

(b) NASA-TLX [72] results on the subscales: mental demand (MD), physical demand (PD), temporal demand (TD), performance (P), effort (E) and frustration (F) split by questionnaire conditions.

Figure 5. Boxplots of presence and workload split by questionnaire type

We indicate how many participants agree with the central statements made by others. Exact quotes were translated by the authors and are labeled with an ID in parentheses.

The majority of the participants $(27 / 38)$ stated their VR experience as "fun" or "enjoyable". 17 found the INVRQS easy to use and the interaction intuitive. 4 participants preferred the directness of input with the VR controllers. However, 16 reported that sometimes the UI did not respond to their input; 4 described this as frustrating. 26 referred to the OUTVRQS as the "common" and "normal" questionnaires. 4 participants reported higher effort using the mouse and 12 participants would have preferred a faster mouse movement in OUTVRQ. Although the participants highlighted the advantages of familiarity using the mouse, we observed that all users instinctively understood the pointing interaction.

17 users criticized changing from VR to physical reality and stated that not having to switch the system feels more fluent and "better integrated" (P12). 4 users addressed the BIP due to the change of medium and how it might affect data quality.

Regarding presentation, 31 participants confirmed that readability and font size of the INVRQS were good and the questionnaire canvas was positioned well. P34 disliked not having a full overview of the displayed content in contrast to the notebook screen. 13 users mentioned that blurred edges in the HMD required head movement for reading. 8 users found it strenuous to fill out the questionnaires in VR. 5 of them suggested to provide a chair, P38 sat on the floor. Concerning data validity, 4 participants hypothesized that maintaining the immersion is "better for the results" (P11). When answering how they feel, 14 participants found it beneficial to do so in VR, because they still were in the situation: "You don't need to recall how you just felt." (P9). Correspondingly, 8 valued the immediacy of the surveying in VR.

12 stated the INVRQS were entertaining: "I didn't know filling out questionnaires can be fun!" (P33). Accordingly, OUTVRQS were referred to as "dryer" (P4) or boring by 12 users, and P35 stated that a pleasant setting can be motivating to fill out the survey. At the same time, such positive excitement may influence certain measures (e.g. affect).

Finally, we asked the participants how they would like to answer questionnaires if the they would have to repeat the study. A majority of $31 / 38$ would prefer to do it in VR, 5 on a computer and 2 had ambivalent opinions.

\section{Discussion}

The high ratings on the IPQ [155] with positive differences against neutral on all subscales but REAL, the ratings of the game and the control over the virtual bow, as well as the qualitative statements indicate that the game provided a high sense of presence. This indicates that our experiment design, which intended to simulate a realistic VR user study scenario, was successful. In alignment with results by Schwind et al. [159], we could not find any differences between the conditions on presence. However, our data show no differences in consistency and we cannot confirm their findings of lower variances of presence when surveying in VR. This supports prior findings that presence questionnaires are inadequate to assess BIPs [171]. Further, literature suggests that presence should be assessed behaviourally $[48,166,172]$ or physiologically $[24,39,116,170]$.

With the UMUX, we measured high usability scores for both questionnaire tools (INVRQ: $M=77.35, S D=18.35$, OUTVRQ: $M=86.21, S D=9.4)$. However, the OUTVRQS were rated higher with a medium effect size (Cohen's $d=.60$ ). A possible explanation was given in the interviews: The participants sometimes had to repeatedly click on a UI element of the INVRQ for selection. The UMUX score allows a comparison with the SUS [46]. According to Bangor the scores are in a highly acceptable range [14], discarding the concerns of the experts. Similarly on the TLX, we measured a comparatively low workload [65]. However, the physical demand and effort are significantly higher in the INVRQ condition. As 5 participants stated, this could be attributed to the fact that they were standing and using the VR controller in mid-air rather than sitting on a chair and using the mouse with a resting hand on 
the desk. The comparable duration is consistent with the TLX ratings for temporal demand and allows to infer that answering questions in VR does not affect the duration of a user study.

\section{Limitations and Future Work}

Our research only investigates the design aspects of the anchoring and interaction modality with INVRQs. This first step was essential to establish a convenient INVRQ design that can be applied in VR user research. Due to small sample size and non-diverse participants in the pre-study our design recommendations are not conclusive. However, the high UMUX scores in the user study confirm the prior results. In future work, we aim to systematically examine further design options (e.g. HUDs, freehand interaction) at different degrees of contextualization and embeddedness as previous work showed for games [53,169] and VR [52, 198].

We only considered a short assessment of an INVRQ $(\approx 6.5 \mathrm{~min})$ at the end of 1 exposure. Future work should examine the effects of long VR exposures and the effect of INVRQS for repeated between-trials measurements. We also did not evaluate open-ended questions which allow the subjects to respond freely, but require sophisticated methods for text-entry in VR. Although such methods exist [92, 129], they are often less accurate and efficient than out-VR settings, especially for untrained users [92]. In future work, we aim to investigate text-entry and oral assessment of open-ended questions in VR as [37], [121] and 4 surveyed experts suggested. The slightly lower UMUX rating suggests room for improvements for the in-VR questionnaire components and the increased TLX physical demand and effort, together with participant comments and behaviour suggest that investigations into "middle-ground" approaches (e.g. remaining in VR but being seated) could lead to more practical solutions.

\section{CONCLUSION}

Subjective self-reports are frequently used in VR user studies and administered in the physical domain. This can lead to a break in presence [83], disrupt the immersive experience [91] and bias the responses [159]. Embedded questionnaires in the VE reinforce the association of VR and the subjective responses. Although different presentation methods of the questionnaires may affect the results, contemporary research has no shared agreement or validated assessment methods of self-reports in VR user studies. This work aggregates the contemporary body of research, VR expert perspectives and the user experience of INVRQS.

Our first research question (RQ1) investigates current applications of questionnaires in VR user research. From the literature analysis, we identified 15 instances of INVRQs. These few examples differ substantially in visualization and interaction, emphasizing the lack of validated surveying procedures in VR user research. For a comprehensive understanding of the advantages and challenges of INVRQS (RQ2), we conducted an online survey with $67 \mathrm{VR}$ experts. 43/67 of researchers see the importance of embedding questionnaires directly into the VE. To explore presentation and interaction modalities suitable for INVRQS (RQ3), we conducted 2 user studies, in which we first identified world-anchoring and pointing as most adequate design choices to administer questionnaires in VR and then contrasted an INVRQ against a common screenbased OUTVRQ. Although the results show lower usability and higher physical demand and effort of INVRQs, the ratings are within tolerable range and the majority of participants stated a positive attitude towards INVRQ.

24/67 of the experts rated the usefulness of INVRQs below neutral and raised concerns regarding usability (10) and the required time for answering (5). The high UMUX-scores and comparable completion time between INVRQS and OUTVRQS defy these objections. Moreover, the majority of the participants $(31 / 38)$ would choose INVRQs over OUTVRQs. This result may be partially attributed to a novelty effect of VR and might weaken with a wider dissemination of VR technology. Similarly, our online survey showed that 29/67 of the experts have already applied INVRQs in user studies and mainly consider them as useful and effective. Based on our findings from the literature review, the survey and the user studies, we advocate that presenting questionnaires in the VE helps the participants to report their experience in a convenient, non-interruptive manner.

Based on the results from the 4 presented studies we conclude: (i) researchers should to apply INVRQs in their user studies, (ii) pointing and world-anchoring are usable ways to realise INVRQS, (iii) participants prefer using INVRQS over OUTVRQS and (iv) researcher should be aware of slightly raised physical and mental demands when using INVRQs.

Similar to the establishment of standardized questionnaires that are empirically validated, we propose moving towards standardizing questionnaire implementation and presentation methods in VR, similar to a quasi-standardization that has already occurred in more traditional screen-based questionnaires due to the prevalence of selected survey tools, such as Google Forms or LimeSurvey [57]. Future work will need to seek for comparative experimental evidence on how the questionnaire modality affects the reliability of the the measurements. As with most design choices, there is no absolute right or wrong. However, researchers should be aware how their measurement methods influence the data. This research lays the groundwork for a design theory of INVRQS to provide validated and standardized methods of question-asking in VR.

\section{ACKNOWLEDGEMENTS}

This project was partly funded by the European Unions' Horizon 2020 research and innovation program (No 688244), project first.stage, Klaus Tschira Stiftung gGmbH, and by the German Research Foundation DFG, as part of Collaborative Research Center (Sonderforschungsbereich) 1320 "EASE - Everyday Activity Science and Engineering", University of Bremen (https://ease-crc.org/). The research was conducted in subproject P01 Embodied semantics for the language of action and change.

\section{REFERENCES}

[1] Ethel Mary Abernethy. 1940. The Effect of Changed Environmental Conditions Upon the Results of College Examinations. The Journal of Psychology 10, 2 (Oct. 
1940), 293-301. DOI :

http://dx.doi.org/10.1080/00223980.1940.9917005

[2] Parastoo Abtahi, Mar Gonzalez-Franco, Eyal Ofek, and Anthony Steed. 2019a. I'm a Giant: Walking in Large Virtual Environments at High Speed Gains. In Proceedings of the 2019 CHI Conference on Human Factors in Computing Systems (CHI '19). ACM, Glasgow, Scotland Uk, 522:1-522:13. DOI :

http://dx.doi.org/10/gf3kzd

[3] Parastoo Abtahi, Benoit Landry, Jackie (Junrui) Yang, Marco Pavone, Sean Follmer, and James A. Landay. 2019b. Beyond The Force: Using Quadcopters to Appropriate Objects and the Environment for Haptics in Virtual Reality. In Proceedings of the 2019 CHI Conference on Human Factors in Computing Systems (CHI '19). ACM, Glasgow, Scotland Uk, 359:1-359:13. DOI : http://dx.doi.org/10/gf3krq

[4] Lu Ann Aday, Llewellyn J. Cornelius, Foreword Steven, B. Cohen, Lu Ann Aday, Llewellyn J. Cornelius, Foreword Steven, B. Cohen, and A. Wiley Imprint. 2006. Designing and Conducting Health Surveys: A Comprehensive Guide (thrid edition ed.). Jossey-Bass Publishers.

[5] G. Ahamba, D. J. Roberts, and P. Eachus. 2017. Triggering and Measuring Social Inhibitory Response in Humans Immersed in Interactions with Virtual Humans. Annual Review of Cybertherapy and Telemedicine 15 (April 2017), 185-188.

[6] Majed Al Zayer, Isayas B. Adhanom, Paul MacNeilage, and Eelke Folmer. 2019. The Effect of Field-of-View Restriction on Sex Bias in VR Sickness and Spatial Navigation Performance. In Proceedings of the 2019 CHI Conference on Human Factors in Computing Systems (CHI '19). ACM, Glasgow, Scotland Uk, 354:1-354:12. DOI : http://dx. doi.org/10/gf3krr

[7] Judith Amores, Xavier Benavides, and Pattie Maes. 2015. ShowMe: A Remote Collaboration System That Supports Immersive Gestural Communication. In Proceedings of the 33rd Annual ACM Conference Extended Abstracts on Human Factors in Computing Systems (CHI EA '15). ACM, New York, NY, USA, 1343-1348. DOI :

http://dx.doi.org/10.1145/2702613.2732927

[8] I. M. Arafat, S. M. Shahnewaz Ferdous, and J. Quarles. 2018. Cybersickness-Provoking Virtual Reality Alters Brain Signals of Persons with Multiple Sclerosis. In 2018 IEEE Conference on Virtual Reality and 3D User Interfaces (VR). 1-120. DOI :

http://dx.doi.org/10/gf39qg

[9] Rahul Arora, Rubaiat Habib Kazi, Fraser Anderson, Tovi Grossman, Karan Singh, and George Fitzmaurice. 2017. Experimental Evaluation of Sketching on Surfaces in VR. ACM Press, 5643-5654. DOI : http://dx.doi.org/10.1145/3025453.3025474

[10] Association for Computing Machinery. 2019. ACM Digital Library. https://dl.acm.org/. (2019).
[11] Mahdi Azmandian, Timofey Grechkin, and Evan Suma Rosenberg. 2017. An Evaluation of Strategies for Two-User Redirected Walking in Shared Physical Spaces. IEEE, 91-98. D0I :

http://dx.doi.org/10.1109/VR.2017.7892235

[12] Earl Babbie. 1990. Survey Research Methods (2nd ed. ed.). Wadsworth, Balmont, Calif.

[13] Chris Baker, Nancy Pistrang, and Robert Elliott. 2002. Research Methods in Clinical Psychology: An Introduction for Students and Practitioners (second edition ed.). John Wiley \& Sons Ltd.

[14] Aaron Bangor, Kortum, Philip, and Miller, James. 2009. Determining What Individual SUS Scores Mean: Adding an Adjective Rating Scale. Journal of usability studies 4, 3 (2009), 114-123.

[15] Soumya C. Barathi, Daniel J. Finnegan, Matthew Farrow, Alexander Whaley, Pippa Heath, Jude Buckley, Peter W. Dowrick, Burkhard C. Wuensche, James L. J. Bilzon, Eamonn O’Neill, and Christof Lutteroth. 2018. Interactive Feedforward for Improving Performance and Maintaining Intrinsic Motivation in VR Exergaming. In Proceedings of the $2018 \mathrm{CHI}$ Conference on Human Factors in Computing Systems (CHI '18). ACM, New York, NY, USA, 408:1-408:14. DOI : http://dx.doi .org/10.1145/3173574.3173982

[16] Jiwan Bhandari, Sam Tregillus, and Eelke Folmer. 2017. Legomotion: Scalable Walking-Based Virtual Locomotion. ACM Press, 1-8. DOI : http://dx.doi.org/10.1145/3139131.3139133

[17] Anol Bhattacherjee. 2012. Social Science Research: Principles, Methods, and Practices (second edition ed.). Number 3 in Textbooks Collection.

[18] Sam B Bhayani and Gerald L Andriole. 2005. Three-Dimensional (3D) Vision: Does It Improve Laparoscopic Skills? An Assessment of a 3D Head-Mounted Visualization System. Reviews in Urology 7, 4 (2005), 211-214.

[19] Bobby Bodenheimer, Sarah Creem-Regehr, Jeanine Stefanucci, Elena Shemetova, and William B.

Thompson. 2017. Prism Aftereffects for Throwing with a Self-Avatar in an Immersive Virtual Environment. IEEE, 141-147. DOI : http://dx.doi.org/10.1109/VR.2017.7892241

[20] M. Boldt, M. Bonfert, I. Lehne, M. Cahnbley, K. Korschinq, L. Bikas, S. Finke, M. Hanci, V. Kraft, B. Liu, T. Nguyen, A. Panova, R. Singh, A. Steenbergen, R. Malaka, and J. Jan Smeddinck. 2018. You Shall Not Pass: Non-Intrusive Feedback for Virtual Walls in VR Environments with Room-Scale Mapping. In 2018 IEEE Conference on Virtual Reality and $3 D$ User Interfaces (VR). 143-150. DOI:

http://dx.doi.org/10.1109/VR.2018.8446177

[21] Stéphane Bouchard, Julie St-Jacques, Geneviève Robillard, and Patrice Renaud. 2008. Anxiety Increases 
the Feeling of Presence in Virtual Reality. Presence: Teleoperators and Virtual Environments 17, 4 (July 2008), 376-391. D0I : http://dx.doi.org/10/bctvw8

[22] LouAnne E. Boyd, Saumya Gupta, Sagar B. Vikmani, Carlos M. Gutierrez, Junxiang Yang, Erik Linstead, and Gillian R. Hayes. 2018. vrSocial: Toward Immersive Therapeutic VR Systems for Children with Autism. In Proceedings of the 2018 CHI Conference on Human Factors in Computing Systems (CHI '18). ACM, New York, NY, USA, 204:1-204:12. DOI : http://dx.doi.org/10.1145/3173574.3173778

[23] Norman M. Bradburn and Seymour Sudman. 1979. Improving Interview Methods and Questionnaire Design - Response Effects to Threatening Qurstions in Survey Research. Jossey-Bass Publishers.

[24] Andrea Brogni, Vinoba Vinayagamoorthy, Anthony Steed, and Mel Slater. 2006. Variations in Physiological Responses of Participants During Different Stages of an Immersive Virtual Environment Experiment. In Proceedings of the ACM Symposium on Virtual Reality Software and Technology (VRST '06). ACM, Limassol, Cyprus, 376-382. DOI : http://dx.doi.org/10/b9drht

[25] John Brooke. 1996. SUS - A Quick and Dirty Usability Scale. (1996), 7.

[26] Emily Brown and Paul Cairns. 2004. A Grounded Investigation of Game Immersion. In Extended Abstracts of the 2004 Conference on Human Factors and Computing Systems - CHI '04. ACM Press, Vienna, Austria, 1297. DOI : http://dx.doi.org/10.1145/985921.986048

[27] Peter W. S. Butcher, Nigel W. John, and Panagiotis D. Ritsos. 2019. VRIA - A Framework for Immersive Analytics on the Web. In Extended Abstracts of the 2019 CHI Conference on Human Factors in Computing Systems (CHI EA '19). ACM, Glasgow, Scotland Uk, LBW2615:1-LBW2615:6. D0I :

http://dx.doi.org/10/gf3kb8

[28] Zekun Cao, Jason Jerald, and Regis Kopper. 2018. Visually-Induced Motion Sickness Reduction via Static and Dynamic Rest Frames. In 2018 IEEE Conference on Virtual Reality and 3D User Interfaces (VR). 105-112. DOI : http://dx.doi .org/10/gf39qh

[29] Sibo Chen, Junce Wang, Santiago Guerra, Neha Mittal, and Soravis Prakkamakul. 2019. Exploring Word-Gesture Text Entry Techniques in Virtual Reality. In Extended Abstracts of the 2019 CHI Conference on Human Factors in Computing Systems (CHI EA '19). ACM, Glasgow, Scotland Uk, LBW0233:1-LBW0233:6. D0I : http://dx.doi.org/10/gf3kb4

[30] Alan Cheng, Lei Yang, and Erik Andersen. 2017b. Teaching Language and Culture with a Virtual Reality Game. In Proceedings of the 2017 CHI Conference on Human Factors in Computing Systems. ACM, 541-549. DOI : http://dx.doi .org/10/gf78wz
[31] Lung-Pan Cheng, Eyal Ofek, Christian Holz, Hrvoje Benko, and Andrew D. Wilson. 2017a. Sparse Haptic Proxy: Touch Feedback in Virtual Environments Using a General Passive Prop. ACM Press, 3718-3728. DOI : http://dx.doi.org/10.1145/3025453.3025753

[32] Bernard C.K. Choi and Anita W.P. Pak. 2004. A Catalog of Biases in Questionnaires. Preventing Chronic Disease 2, 1 (Dec. 2004).

[33] Tanvir Irfan Chowdhury, Sharif Mohammad Shahnewaz Ferdous, and John Quarles. 2017. Information Recall in a Virtual Reality Disability Simulation. ACM Press, 1-10. DOI : http://dx.doi.org/10.1145/3139131.3139143

[34] Sebastian Cmentowski, Andrey Krekhov, and Jens Krueger. 2019. Outstanding: A Perspective-Switching Technique for Covering Large Distances in VR Games. In Extended Abstracts of the 2019 CHI Conference on Human Factors in Computing Systems (CHI EA '19). ACM, Glasgow, Scotland Uk, LBW1612:1-LBW1612:6. DOI : http://dx.doi.org/10/gf3kcd

[35] Mihaly Csikszentmihalyi. 1990. Flow: The Psychology of Optimal Experience. Harper \& Row, New York.

[36] Lynnda M. Dahlquist, Kristine D. McKenna, Katia K. Jones, Lindsay Dillinger, Karen E. Weiss, and Claire Sonntag Ackerman. 2007. Active and Passive Distraction Using a Head-Mounted Display Helmet: Effects on Cold Pressor Pain in Children. Health Psychology: Official Journal of the Division of Health Psychology, American Psychological Association 26, 6 (Nov. 2007), 794-801. DOI :

http://dx.doi.org/10/cmp2vx

[37] Donald Degraen, André Zenner, and Antonio Krüger. 2019. Enhancing Texture Perception in Virtual Reality Using 3D-Printed Hair Structures. In Proceedings of the 2019 CHI Conference on Human Factors in Computing Systems (CHI '19). ACM, Glasgow, Scotland Uk, 249:1-249:12. DOI :

http://dx.doi.org/10/gfz8mf

[38] Arindam Dey, Hao Chen, Mark Billinghurst, and Robert W. Lindeman. 2018. Effects of Manipulating Physiological Feedback in Immersive Virtual Environments. In Proceedings of the 2018 Annual Symposium on Computer-Human Interaction in Play (CHI PLAY '18). ACM, Melbourne, VIC, Australia, 101-111. DOI : http://dx. doi .org/10/gf3ngn

[39] Cath Dillon, Edmund Keogh, and Jonathan Freeman. 2002. It's Been Emotional: Affect, Physiology, and Presence. In Proceedings of the Fifth Annual International Workshop on Presence, Porto, Portugal.

[40] Tilman Dingler, Kai Kunze, and Benjamin Outram. 2018. VR Reading UIs: Assessing Text Parameters for Reading in VR. In Extended Abstracts of the 2018 CHI Conference on Human Factors in Computing Systems (CHI EA '18). ACM, New York, NY, USA, 
LBW094:1-LBW094:6. D0I :

http://dx.doi.org/10.1145/3170427.3188695

[41] Alan Dix. 2009. Human-Computer Interaction. In Encyclopedia of Database Systems. Springer, 1327-1331.

[42] E. Ebrahimi, L. S. Hartman, A. Robb, C. C. Pagano, and S. V. Babu. 2018. Investigating the Effects of Anthropomorphic Fidelity of Self-Avatars on Near Field Depth Perception in Immersive Virtual Environments. In 2018 IEEE Conference on Virtual Reality and $3 D$ User Interfaces (VR). 1-8. DOI : http://dx.doi.org/10/gf39qs

[43] Claudia Faita, Federico Vanni, Camilla Tanca, Emanuele Ruffaldi, Marcello Carrozzino, and Massimo Bergamasco. 2016. Investigating the Process of Emotion Recognition in Immersive and Non-Immersive Virtual Technological Setups. ACM Press, 61-64. D0I : http://dx.doi.org/10.1145/2993369.2993395

[44] A. S. Fernandes and S. K. Feiner. 2016. Combating VR Sickness through Subtle Dynamic Field-of-View Modification. In 2016 IEEE Symposium on 3D User Interfaces (3DUI). 201-210. DOI: http://dx.doi.org/10/gcmfn6

[45] Andy Field and Graham Hole. 2003. How to Design and Report Experiments. Sage Publications Ltd, London ; Thousand Oaks, Calif.

[46] Kraig Finstad. 2010. The Usability Metric for User Experience. Interacting with Computers 22, 5 (Sept. 2010), 323-327. DOI : http://dx.doi.org/10/ctr6r4

[47] Daniel Freeman, Polly Haselton, Jason Freeman, Bernhard Spanlang, Sameer Kishore, Emily Albery, Megan Denne, Poppy Brown, Mel Slater, and Alecia Nickless. 2018. Automated Psychological Therapy Using Immersive Virtual Reality for Treatment of Fear of Heights: A Single-Blind, Parallel-Group, Randomised Controlled Trial. The Lancet Psychiatry 5, 8 (Aug. 2018), 625-632. DOI :

http://dx.doi.org/10/gd2h5n

[48] Jonathan Freeman, S. E. Avons, Ray Meddis, Don E. Pearson, and Wijnand IJsselsteijn. 2000. Using Behavioral Realism to Estimate Presence: A Study of the Utility of Postural Responses to Motion Stimuli. Presence: Teleoperators and Virtual Environments 9, 2 (April 2000), 149-164. DOI :

http://dx.doi.org/10/drkrr8

[49] Jonathan Freeman, S. E. Avons, Don E. Pearson, and Wijnand A. IJsselsteijn. 1999. Effects of Sensory Information and Prior Experience on Direct Subjective Ratings of Presence. Presence: Teleoperators and Virtual Environments 8, 1 (Feb. 1999), 1-13. D0I: http://dx. doi .org/10/dg7fn6

[50] R. Fribourg, F. Argelaguet, L. Hoyet, and A. Lécuyer. 2018. Studying the Sense of Embodiment in VR
Shared Experiences. In 2018 IEEE Conference on Virtual Reality and 3D User Interfaces (VR). 273-280. DOI : http://dx.doi .org/10/gf39p6

[51] Thomas Fröhlich, Dmitry Alexandrovsky, Timo Stabbert, Tanja Döring, and Rainer Malaka. 2018. VRBox: A Virtual Reality Augmented Sandbox for Immersive Playfulness, Creativity and Exploration. In Proceedings of the 2018 Annual Symposium on Computer-Human Interaction in Play (CHI PLAY '18). ACM, Melbourne, VIC, Australia, 153-162. DOI : http://dx.doi.org/10/gfsvjx

[52] Julian Frommel, Kim Fahlbusch, Julia Brich, and Michael Weber. 2017. The Effects of Context-Sensitive Tutorials in Virtual Reality Games. ACM Press, 367-375. DOI :

http://dx.doi.org/10.1145/3116595.3116610

[53] Julian Frommel, Michael Weber, Katja Rogers, Julia Brich, Daniel Besserer, Leonard Bradatsch, Isabel Ortinau, Ramona Schabenberger, Valentin Riemer, and Claudia Schrader. 2015. Integrated Questionnaires: Maintaining Presence in Game Environments for Self-Reported Data Acquisition. In Proceedings of the 2015 Annual Symposium on Computer-Human Interaction in Play - CHI PLAY'15. ACM Press, London, United Kingdom, 359-368. DOI: http://dx.doi.org/10/gfvjmx

[54] Wei Gai, Cheng Lin, Chenglei Yang, Yulong Bian, Chia Shen, Xiangxu Meng, Lu Wang, Juan Liu, Mingda Dong, and Chengjie Niu. 2017. Supporting Easy Physical-to-Virtual Creation of Mobile VR Maze Games: A New Genre. ACM Press, 5016-5028. DOI : http://dx.doi.org/10.1145/3025453.3025494

[55] D. Gall and M. E. Latoschik. 2018. The Effect of Haptic Prediction Accuracy on Presence. In 2018 IEEE Conference on Virtual Reality and $3 D$ User Interfaces (VR). 73-80. DOI : http://dx.doi.org/10/gf39qm

[56] Maryrose Gerardi, Barbara Olasov Rothbaum, Kerry Ressler, Mary Heekin, and Albert Rizzo. 2008. Virtual reality exposure therapy using a virtual Iraq: Case report. Journal of Traumatic Stress 21, 2 (April 2008), 209-213. DOI : http://dx.doi.org/10/djjmn5

[57] Limesurvey GmbH. 2003. LimeSurvey: An Open Source Survey Tool. (2003).

[58] D. R. Godden and A. D. Baddeley. 1975. Context-Dependent MemoryIn Two Natural Environments: On Land and Underwater. British Journal of Psychology 66, 3 (Aug. 1975), 325-331. DOI : http://dx.doi.org/10/cjgxhp

[59] David Goedicke, Jamy Li, Vanessa Evers, and Wendy Ju. 2018. VR-OOM: Virtual Reality On-rOad Driving siMulation. In Proceedings of the $2018 \mathrm{CHI}$ Conference on Human Factors in Computing Systems (CHI '18). ACM, New York, NY, USA, 165:1-165:11. DOI : http://dx.doi.org/10.1145/3173574.3173739 
[60] Jeffrey I. Gold, Seok Hyeon Kim, Alexis J. Kant, Michael H. Joseph, and Albert "Skip" Rizzo. 2006. Effectiveness of Virtual Reality for Pediatric Pain Distraction during IV Placement. CyberPsychology \& Behavior 9, 2 (April 2006), 207-212. DOI : http://dx.doi.org/10/fwsrpx

[61] Google. 2018. Rendering Text in WebVR I Web. https://developers.google.com/web/showcase/2017/within. (Sept. 2018).

[62] Google Developers. 2017. Designing Screen Interfaces for VR (Google I/O'17). (2017).

[63] Antti Granqvist, Tapio Takala, Jari Takatalo, and Perttu Hämäläinen. 2018. Exaggeration of Avatar Flexibility in Virtual Reality. In Proceedings of the 2018 Annual Symposium on Computer-Human Interaction in Play (CHI PLAY '18). ACM, Melbourne, VIC, Australia, 201-209. DOI : http://dx.doi.org/10/gfvpff

[64] Anthony G. Greenwald and Brian A. Nosek. 2001. Health of the Implicit Association Test at Age 3. Experimental Psychology 48, 2 (April 2001), 85-93. DOI : http://dx.doi.org/10/c4k2qx

[65] Rebecca A. Grier. 2015. How High Is High? A Meta-Analysis of NASA-TLX Global Workload Scores. Proceedings of the Human Factors and Ergonomics Society Annual Meeting 59, 1 (Sept. 2015), 1727-1731. DOI : http://dx.doi.org/10/gf447w

[66] Nathan Navarro Griffin, James Liu, and Eelke Folmer. 2018. Evaluation of Handsbusy vs Handsfree Virtual Locomotion. In Proceedings of the 2018 Annual Symposium on Computer-Human Interaction in Play (CHI PLAY '18). ACM, Melbourne, VIC, Australia, 211-219. DOI : http://dx.doi.org/10/gf3ngm

[67] J. Grubert, L. Witzani, E. Ofek, M. Pahud, M. Kranz, and P. O. Kristensson. 2018a. Effects of Hand Representations for Typing in Virtual Reality. In 2018 IEEE Conference on Virtual Reality and $3 D$ User Interfaces (VR). 151-158. DOI: http://dx.doi.org/10/gf39qd

[68] J. Grubert, L. Witzani, E. Ofek, M. Pahud, M. Kranz, and P. O. Kristensson. 2018b. Text Entry in Immersive Head-Mounted Display-Based Virtual Reality Using Standard Keyboards. In 2018 IEEE Conference on Virtual Reality and 3D User Interfaces (VR). 159-166. DOI : http://dx.doi.org/10/gf39qc

[69] Jan Gugenheimer, Evgeny Stemasov, Julian Frommel, and Enrico Rukzio. 2017. ShareVR: Enabling Co-Located Experiences for Virtual Reality between HMD and Non-HMD Users. ACM Press, 4021-4033. DOI : http://dx.doi.org/10.1145/3025453.3025683

[70] Jan Gugenheimer, Evgeny Stemasov, Harpreet Sareen, and Enrico Rukzio. 2018. FaceDisplay: Towards Asymmetric Multi-User Interaction for Nomadic Virtual Reality. In Proceedings of the $2018 \mathrm{CHI}$ Conference on Human Factors in Computing Systems
(CHI '18). ACM, New York, NY, USA, 54:1-54:13. DOI : http://dx.doi .org/10.1145/3173574. 3173628

[71] John Paulin Hansen, Astrid Kofod Trudslev, Sara Amdi Harild, Alexandre Alapetite, and Katsumi Minakata. 2019. Providing Access to VR Through a Wheelchair. In Extended Abstracts of the 2019 CHI Conference on Human Factors in Computing Systems (CHI EA '19). ACM, Glasgow, Scotland Uk, CS07:1-CS07:8. DOI : http://dx. doi .org/10/gf3kb2

[72] Sandra G. Hart. 1986. NASA Task Load Index (TLX). Volume 1.0; Paper and Pencil Package. Technical Report.

[73] Jeremy Hartmann, Christian Holz, Eyal Ofek, and Andrew D. Wilson. 2019. RealityCheck: Blending Virtual Environments with Situated Physical Reality. In Proceedings of the 2019 CHI Conference on Human Factors in Computing Systems (CHI '19). ACM, Glasgow, Scotland Uk, 347:1-347:12. DOI : http://dx.doi.org/10/gf2hj4

[74] Tianyu He, Xiaoming Chen, Zhibo Chen, Ye Li, Sen Liu, Junhui Hou, and Ying He. 2017. Immersive and Collaborative Taichi Motion Learning in Various VR Environments. IEEE, 307-308. DOI : http://dx.doi.org/10.1109/VR.2017.7892299

[75] Teresa Hirzle, Jan Rixen, Jan Gugenheimer, and Enrico Rukzio. 2018. WatchVR: Exploring the Usage of a Smartwatch for Interaction in Mobile Virtual Reality. In Extended Abstracts of the 2018 CHI Conference on Human Factors in Computing Systems (CHI EA '18). ACM, New York, NY, USA, LBW634:1-LBW634:6. DOI : http://dx.doi .org/10.1145/3170427.3188629

[76] Philipp Hock, Sebastian Benedikter, Jan Gugenheimer, and Enrico Rukzio. 2017. CarVR: Enabling In-Car Virtual Reality Entertainment. ACM Press, 4034-4044. DOI : http://dx.doi .org/10.1145/3025453.3025665

[77] Matthias Hoppe, Jakob Karolus, Felix Dietz, PawełW. Woźniak, Albrecht Schmidt, and Tonja-Katrin Machulla. 2019. VRsneaky: Increasing Presence in VR Through Gait-Aware Auditory Feedback. In Proceedings of the 2019 CHI Conference on Human Factors in Computing Systems (CHI'19). ACM, Glasgow, Scotland Uk, 546:1-546:9. DOI: http://dx.doi.org/10/gf3kzc

[78] HTC. 2016. VIVE Now Shipping Immediately From HTC, Retail Partners Expand Demo Locations. https://www.htc.com/us/newsroom/2016-06-07/. (2016).

[79] Brent E. Insko. 2003. Measuring Presence: Subjective, Behavioral and Physiological Methods. In Being There: Concepts, Effects and Measurements of User Presence in Synthetic Environments. IOS Press, Amsterdam, Netherlands, 109-119.

[80] Institute of Electrical and Electronics Engineers. 2019. IEEE Xplore Digital Library. https://ieeexplore.ieee.org/. (2019). 
[81] International Organization for Standardization (ISO). 2006. Ergonomics of Human-System Interaction Part 110: Dialogue Principles (ISO 9241-110). (2006).

[82] Charlene Jennett, Anna L. Cox, Paul Cairns, Samira Dhoparee, Andrew Epps, Tim Tijs, and Alison Walton. 2008. Measuring and Defining the Experience of Immersion in Games. International Journal of Human-Computer Studies 66, 9 (Sept. 2008), 641-661. DOI : http://dx.doi .org/10/ct9vk5

[83] Jason Jerald. 2016. The VR Book: Human-Centered Design for Virtual Reality (first edition ed.). Number 8 in ACM Books. acm, Association for Computing Machinery, New York. OCLC: 945367096.

[84] S. Jung, P. J. Wisniewski, and C. E. Hughes. 2018. In Limbo: The Effect of Gradual Visual Transition Between Real and Virtual on Virtual Body Ownership Illusion and Presence. In 2018 IEEE Conference on Virtual Reality and 3D User Interfaces (VR). 267-272. DOI : http://dx. doi .org/10/gf39p7

[85] Hyeong Yeop Kang, Geonsun Lee, Seongsu Kwon, Ohung Kwon, Seongpil Kim, and JungHyun Han. 2018. Flotation Simulation in a Cable-Driven Virtual Environment - A Study with Parasailing. In Proceedings of the 2018 CHI Conference on Human Factors in Computing Systems (CHI'18). ACM, New York, NY, USA, 632:1-632:11. DOI : http://dx. doi .org/10.1145/3173574.3174206

[86] Oliver Beren Kaul and Michael Rohs. 2017. HapticHead: A Spherical Vibrotactile Grid around the Head for 3D Guidance in Virtual and Augmented Reality. ACM Press, 3729-3740. DOI : http://dx.doi.org/10.1145/3025453.3025684

[87] Robert S. Kennedy, Norman E. Lane, Kevin S. Berbaum, and Michael G. Lilienthal. 1993. Simulator Sickness Questionnaire: An Enhanced Method for Quantifying Simulator Sickness. The International Journal of Aviation Psychology 3, 3 (July 1993), 203-220. DOI : http://dx.doi .org/10/bbh54v

[88] Behrang Keshavarz and Heiko Hecht. 2011. Validating an Efficient Method to Quantify Motion Sickness. Human Factors 53, 4 (Aug. 2011), 415-426. DOI : http://dx.doi.org/10/dz78cg

[89] Youngwon Ryan Kim and Gerard J. Kim. 2017. Presence and Immersion of "Easy" Mobile VR with Open Flip-on Lenses. In Proceedings of the 23rd ACM Symposium on Virtual Reality Software and Technology (VRST'17). ACM, New York, NY, USA, 38:1-38:7. DOI : http://dx.doi .org/10.1145/3139131.3139147

[90] Tina Kjær, Christoffer B. Lillelund, Mie Moth-Poulsen, Niels C. Nilsson, Rolf Nordahl, and Stefania Serafin. 2017. Can You Cut It?: An Exploration of the Effects of Editing in Cinematic Virtual Reality. ACM Press, 1-4. DOI : http://dx. doi .org/10.1145/3139131. 3139166

[91] Jarrod Knibbe, Jonas Schjerlund, Mathias Petraeus, and Kasper Hornb $\backslash\{$ ae $\}$ k. 2018. The Dream Is
Collapsing: The Experience of Exiting VR. In Proceedings of the 2018 CHI Conference on Human Factors in Computing Systems (CHI'18). ACM, New York, NY, USA, 483:1-483:13. DOI :

http://dx.doi.org/10.1145/3173574.3174057

[92] Pascal Knierim, Valentin Schwind, Anna Maria Feit, Florian Nieuwenhuizen, and Niels Henze. 2018. Physical Keyboards in Virtual Reality: Analysis of Typing Performance and Effects of Avatar Hands. In Proceedings of the 2018 CHI Conference on Human Factors in Computing Systems (CHI '18). ACM, New York, NY, USA, 345:1-345:9. DOI : http://dx.doi.org/10.1145/3173574.3173919

[93] Julian Kreimeier and Timo Götzelmann. 2019. First Steps Towards Walk-In-Place Locomotion and Haptic Feedback in Virtual Reality for Visually Impaired. In Extended Abstracts of the 2019 CHI Conference on Human Factors in Computing Systems (CHI EA '19). ACM, Glasgow, Scotland Uk, LBW2214:1-LBW2214:6. DOI : http://dx.doi.org/10/gf3kcb

[94] Andrey Krekhov, Sebastian Cmentowski, Katharina Emmerich, Maic Masuch, and Jens Krüger. 2018. GulliVR: A Walking-Oriented Technique for Navigation in Virtual Reality Games Based on Virtual Body Resizing. In Proceedings of the 2018 Annual Symposium on Computer-Human Interaction in Play (CHI PLAY '18). ACM, Melbourne, VIC, Australia, 243-256. DOI : http://dx.doi.org/10/gf3ngk

[95] Andrey Krekhov, Katharina Emmerich, Philipp Bergmann, Sebastian Cmentowski, and Jens Krüger. 2017. Self-Transforming Controllers for Virtual Reality First Person Shooters. ACM Press, 517-529. DOI : http://dx.doi.org/10.1145/3116595.3116615

[96] Heinz W. Krohne, Boris Egloff, Carl-Walter Kohlmann, and Anja Tausch. 1996. Untersuchungen Mit Einer Deutschen Version Der "Positive and Negative Affect Schedule"(PANAS). Diagnostica-Gottingen- 42 (1996), 139-156.

[97] W. Kruger, C. Bohn, B. Frohlich, H. Schuth, W. Strauss, and G. Wesche. 1995. The Responsive Workbench: A Virtual Work Environment. Computer 28, 7 (July 1995), 42-48. DOI : http://dx.doi.org/10/cvbntz

[98] D. M. Krum, S. Kang, and T. Phan. 2018. Influences on the Elicitation of Interpersonal Space with Virtual Humans. In 2018 IEEE Conference on Virtual Reality and $3 D$ User Interfaces (VR). 223-9. DOI : http://dx.doi.org/10/gf39p8

[99] David M. Krum, Sin-Hwa Kang, Thai Phan, Lauren Cairco Dukes, and Mark Bolas. 2017. Social Impact of Enhanced Gaze Presentation Using Head Mounted Projection. In Proceedings of the Human-Computer Interaction International Conference. Springer International Publishing. 
[100] L. Kruse, E. Langbehn, and F. Stelnlcke. 2018. I Can See on My Feet While Walking: Sensitivity to Translation Gains with Visible Feet. In 2018 IEEE Conference on Virtual Reality and 3D User Interfaces (VR). 305-312. DOI : http://dx. doi .org/10/gf39p4

[101] Jérémy Lacoche, Nico Pallamin, Thomas Boggini, and Jérôme Royan. 2017. Collaborators Awareness for User Cohabitation in Co-Located Collaborative Virtual Environments. ACM Press, 1-9. DOI : http://dx.doi.org/10.1145/3139131.3139142

[102] Joseph J. LaViola, Ernst Kruijff, Doug A. Bowman, Ryan P. McMahan, and Ivan Poupyrev. 2017. 3D User Interfaces: Theory and Practice. Addison-Wesley.

[103] Jonathan Lazar, Jinjuan Heidi Feng, and Harry Hochheiser. 2017. Research Methods in Human-Computer Interaction (2 ed.). Morgan Kaufmann, Cambridge, MA.

[104] Kangdon Lee. 2012. Augmented Reality in Education and Training. TechTrends 56, 2 (March 2012), 13-21. DOI : http://dx.doi .org/10/gdz3kh

[105] Myungho Lee, Gerd Bruder, and Gregory F. Welch. 2017. Exploring the Effect of Vibrotactile Feedback through the Floor on Social Presence in an Immersive Virtual Environment. IEEE, 105-111. DOI : http://dx.doi.org/10.1109/VR.2017.7892237

[106] Meng-Yun Liao, Ching-Ying Sung, Hao-Chuan Wang, Wen-Chieh Lin, and Fu-Yin Cherng. 2019. Embodying Historical Learners' Messages As Learning Companions in a VR Classroom. In Extended Abstracts of the 2019 CHI Conference on Human Factors in Computing Systems (CHI EA '19). ACM, Glasgow, Scotland Uk, LBW1115:1-LBW1115:6. DOI : http://dx.doi.org/10/gf3kcg

[107] Pedro Lopes, Sijing You, Lung-Pan Cheng, Sebastian Marwecki, and Patrick Baudisch. 2017. Providing Haptics to Walls \& Heavy Objects in Virtual Reality by Means of Electrical Muscle Stimulation. ACM Press, 1471-1482. DOI :

http://dx.doi.org/10.1145/3025453.3025600

[108] Sarah Lopez, Yi Yang, Kevin Beltran, Soo Jung Kim, Jennifer Cruz Hernandez, Chelsy Simran, Bingkun Yang, and Beste F. Yuksel. 2019. Investigating Implicit Gender Bias and Embodiment of White Males in Virtual Reality with Full Body Visuomotor Synchrony. In Proceedings of the 2019 CHI Conference on Human Factors in Computing Systems. ACM, 557. D0I: http://dx.doi.org/10/gf3kzb

[109] C. Di Loreto, J. Chardonnet, J. Ryard, and A. Rousseau. 2018. WoaH: A Virtual Reality Work-at-Height Simulator. In 2018 IEEE Conference on Virtual Reality and 3D User Interfaces (VR). 281-288. DOI : http://dx. doi . org/10/gf39p5

[110] J. Lugrin, M. Ertl, P. Krop, R. Klüpfel, S. Stierstorfer, B. Weisz, M. Rück, J. Schmitt, N. Schmidt, and M. E.
Latoschik. 2018. Any "Body" There? Avatar Visibility Effects in a Virtual Reality Game. In 2018 IEEE Conference on Virtual Reality and $3 D$ User Interfaces (VR). 17-24. DOI : http://dx.doi .org/10/gf39qq

[111] Raquel Macedo, Nuno Correia, and Teresa Romão. 2019. Paralympic VR Game: Immersive Game Using Virtual Reality and Video. In Extended Abstracts of the 2019 CHI Conference on Human Factors in Computing Systems (CHI EA '19). ACM, Glasgow, Scotland Uk, LBW0235:1-LBW0235:6. DOI :

http://dx.doi.org/10/gf3kb3

[112] I. Scott MacKenzie, Tatu Kauppinen, and Miika Silfverberg. 2001. Accuracy Measures for Evaluating Computer Pointing Devices. In Proceedings of the SIGCHI Conference on Human Factors in Computing Systems (CHI '01). ACM, Seattle, Washington, USA, 9-16. DOI : http://dx.doi.org/10.1145/365024.365028 MacKenzie2001.

[113] I. Scott MacKenzie, Abigail Sellen, and William A. S. Buxton. 1991. A Comparison of Input Devices in Element Pointing and Dragging Tasks. In Proceedings of the SIGCHI Conference on Human Factors in Computing Systems (CHI '91). ACM, New York, NY, USA, 161-166. DOI :

http://dx.doi.org/10.1145/108844.108868

[114] Elhassan Makled, Yomna Abdelrahman, Noha Mokhtar, Valentin Schwind, Slim Abdennadher, and Albrecht Schmidt. 2018. I Like to Move It: Investigating the Effect of Head and Body Movement of Avatars in VR on User's Perception. In Extended Abstracts of the 2018 CHI Conference on Human Factors in Computing Systems (CHI EA'18). ACM, New York, NY, USA, LBW099:1-LBW099:6. DOI : http://dx.doi.org/10.1145/3170427.3188573

[115] Willie McClinton, Derek Caprio, Denis Laesker, Blanche Pinto, Sarah Garcia, and Marvin Andujar. 2019. P300-Based 3D Brain Painting in Virtual Reality. In Extended Abstracts of the 2019 CHI Conference on Human Factors in Computing Systems (CHI EA '19). ACM, Glasgow, Scotland Uk, LBW1119:1-LBW1119:6. DOI : http://dx.doi.org/10/gf3kcf

[116] Michael Meehan. 2001. Physiological Reaction as an Objective Measure of Presence in Virtual Environments. Ph.D. Dissertation.

[117] Daniel Mendes, Maurício Sousa, Rodrigo Lorena, Alfredo Ferreira, and Joaquim Jorge. 2017. Using Custom Transformation Axes for Mid-Air Manipulation of 3D Virtual Objects. ACM Press, 1-8. DOI : http://dx.doi.org/10.1145/3139131.3139157

[118] Mark R Mine. 1997. ISAAC: A Meta-Cad System for Virtual Environments. Computer-Aided Design 29, 8 (Aug. 1997), 547-553. DOI: http://dx.doi.org/10/b6gzzj 
[119] Thomas B. Moeslund, Moritz Störring, and Erik Granum. 2002. A Natural Interface to a Virtual Environment through Computer Vision-Estimated Pointing Gestures. In Gesture and Sign Language in Human-Computer Interaction (Lecture Notes in Computer Science), Ipke Wachsmuth and Timo Sowa (Eds.). Springer Berlin Heidelberg, 59-63.

[120] Aske Mottelson and Kasper Hornbæk. 2017. Virtual Reality Studies Outside the Laboratory. ACM Press, 1-10. DOI : http://dx.doi .org/10.1145/3139131.3139141

[121] C. Mousas. 2018. Performance-Driven Dance Motion Control of a Virtual Partner Character. In 2018 IEEE Conference on Virtual Reality and $3 D$ User Interfaces (VR). 57-64. DOI : http://dx.doi.org/10/gf39qp

[122] Thomas Muender, Anke V. Reinschluessel, Sean Drewes, Dirk Wenig, Tanja Döring, and Rainer Malaka. 2019. Does It Feel Real?: Using Tangibles with Different Fidelities to Build and Explore Scenes in Virtual Reality. In Proceedings of the 2019 CHI Conference on Human Factors in Computing Systems (CHI '19). ACM, Glasgow, Scotland Uk, 673:1-673:12. DOI : http://dx.doi.org/10/gf2d3x

[123] Marcus R. Munafò, Brian A. Nosek, Dorothy V. M. Bishop, Katherine S. Button, Christopher D. Chambers, Nathalie Percie du Sert, Uri Simonsohn, Eric-Jan Wagenmakers, Jennifer J. Ware, and John P. A. Ioannidis. 2017. A Manifesto for Reproducible Science. Nature Human Behaviour 1, 1 (Jan. 2017), 0021. DOI: http://dx.doi.org/10/bw28

[124] S. Narang, A. Best, and D. Manocha. 2018. Simulating Movement Interactions Between Avatars Agents in Virtual Worlds Using Human Motion Constraints. In 2018 IEEE Conference on Virtual Reality and $3 D$ User Interfaces (VR). 9-16. DOI:

http://dx.doi.org/10/gf39qr

[125] Cuong Nguyen, Stephen DiVerdi, Aaron Hertzmann, and Feng Liu. 2017. Vremiere: In-Headset Virtual Reality Video Editing. ACM Press, 5428-5438. DOI: http://dx.doi.org/10.1145/3025453.3025675

[126] Cuong Nguyen, Stephen DiVerdi, Aaron Hertzmann, and Feng Liu. 2018. Depth Conflict Reduction for Stereo VR Video Interfaces. In Proceedings of the 2018 CHI Conference on Human Factors in Computing Systems (CHI '18). ACM, New York, NY, USA, 64:1-64:9. DOI :

http://dx.doi.org/10.1145/3173574.3173638

[127] Sebastian Oberdörfer, David Heidrich, and Marc Erich Latoschik. 2019. Usability of Gamified Knowledge Learning in VR and Desktop-3D. In Proceedings of the 2019 CHI Conference on Human Factors in Computing Systems (CHI'19). ACM, Glasgow, Scotland Uk, 175:1-175:13. DOI : http://dx.doi .org/10/gf3k86
[128] Oculus. 2019. Introduction to Best Practices. https://developer.oculus.com/design/latest/concepts/book$\mathrm{bp} /$. (Sept. 2019).

[129] Eyal Ofek and Michel Pahud. 2018. Typing in the Virtual Office Environment: Examining the Effects of Keyboard and Hand Representation in VR. (Feb. 2018).

[130] Roshan Lalintha Peiris, Wei Peng, Zikun Chen, Liwei Chan, and Kouta Minamizawa. 2017. ThermoVR: Exploring Integrated Thermal Haptic Feedback with Head Mounted Displays. ACM Press, 5452-5456. DOI: http://dx.doi.org/10.1145/3025453.3025824

[131] Xiaolan Peng, Jin Huang, Linghan Li, Chen Gao, Hui Chen, Feng Tian, and Hongan Wang. 2019. Beyond Horror and Fear: Exploring Player Experience Invoked by Emotional Challenge in VR Games. In Extended Abstracts of the 2019 CHI Conference on Human Factors in Computing Systems (CHI EA '19). ACM, Glasgow, Scotland Uk, LBW1616:1-LBW1616:6. DOI : http://dx.doi .org/10/gf3kcc

[132] Max Pfeiffer, Tobias Kröger, Jens Seifert, Sulaxan Somaskantharajan, Lukas Jahnich, Tobias Steinblum, Jan Speckamp, and Samuel Navas Medrano. 2019. WONDER - Enhancing VR Training with Electrical Muscle Stimulation. In Extended Abstracts of the 2019 CHI Conference on Human Factors in Computing Systems (CHI EA '19). ACM, Glasgow, Scotland Uk, LBW0232:1-LBW0232:6. DOI : http://dx.doi.org/10/gf3kb5

[133] Ken Pfeuffer, Matthias J. Geiger, Sarah Prange, Lukas Mecke, Daniel Buschek, and Florian Alt. 2019. Behavioural Biometrics in VR: Identifying People from Body Motion and Relations in Virtual Reality. In Proceedings of the 2019 CHI Conference on Human Factors in Computing Systems (CHI'19). ACM, Glasgow, Scotland Uk, 110:1-110:12. DOI: http://dx.doi.org/10/gf3kx6

[134] Dario Pittera, Elia Gatti, and Marianna Obrist. 2019. I'm Sensing in the Rain: Spatial Incongruity in Visual-Tactile Mid-Air Stimulation Can Elicit Ownership in VR Users. In Proceedings of the 2019 CHI Conference on Human Factors in Computing Systems (CHI '19). ACM, Glasgow, Scotland Uk, 132:1-132:15. DOI : http://dx.doi.org/10/gf3kx5

[135] Thammathip Piumsomboon, Gun A. Lee, Jonathon D. Hart, Barrett Ens, Robert W. Lindeman, Bruce H. Thomas, and Mark Billinghurst. 2018. Mini-Me: An Adaptive Avatar for Mixed Reality Remote Collaboration. In Proceedings of the $2018 \mathrm{CHI}$ Conference on Human Factors in Computing Systems (CHI '18). ACM, New York, NY, USA, 46:1-46:13. DOI : http://dx.doi.org/10.1145/3173574. 3173620

[136] Rüdiger Pohl and Rüdiger F. Pohl. 2004. Cognitive Illusions: A Handbook on Fallacies and Biases in Thinking, Judgement and Memory. Psychology Press. 
[137] Susanne Putze, Dmitry Alexandrovsky, Felix Putze, Sebastian Höffner, Jan D. Smeddinck, and Rainer Malaka. 2020. Breaking The Experience: Effects of Questionnaires in VR User Studies. In Proceedings of the 2020 CHI Conference on Human Factors in Computing Systems (CHI '20). Honolulu, HI, USA. DOI : http://dx.doi .org/10.1145/3313831.3376144

[138] J. T. Quaglia and A. Holecek. 2018. Lucid Virtual Dreaming: Antecedents and Consequents of Virtual Lucidity During Virtual Threat. In 2018 IEEE Conference on Virtual Reality and $3 D$ User Interfaces (VR). 1-72. DOI : http://dx.doi.org/10/gf39qn

[139] Nimesha Ranasinghe, Pravar Jain, Shienny Karwita, David Tolley, and Ellen Yi-Luen Do. 2017. Ambiotherm: Enhancing Sense of Presence in Virtual Reality by Simulating Real-World Environmental Conditions. ACM Press, 1731-1742. DOI : http://dx.doi.org/10.1145/3025453.3025723

[140] Michael Rietzler, Florian Geiselhart, Julian Frommel, and Enrico Rukzio. 2018. Conveying the Perception of Kinesthetic Feedback in Virtual Reality Using State-of-the-Art Hardware. In Proceedings of the 2018 CHI Conference on Human Factors in Computing Systems (CHI'18). ACM, New York, NY, USA, 460:1-460:13. DOI :

http://dx.doi.org/10.1145/3173574.3174034

[141] Michael Rietzler, Florian Geiselhart, and Enrico Rukzio. 2017. The Matrix Has You: Realizing Slow Motion in Full-Body Virtual Reality. ACM Press, 1-10. DOI : http://dx.doi .org/10.1145/3139131.3139145

[142] Katja Rogers, Jana Funke, Julian Frommel, Sven Stamm, and Michael Weber. 2019. Exploring Interaction Fidelity in Virtual Reality: Object Manipulation and Whole-Body Movements. In Proceedings of the 2019 CHI Conference on Human Factors in Computing Systems (CHI'19). ACM, Glasgow, Scotland Uk, 414:1-414:14. DOI : http://dx.doi.org/10/gf2hj3

[143] Katja Rogers, Giovanni Ribeiro, Rina R. Wehbe, Michael Weber, and Lennart E. Nacke. 2018. Vanishing Importance: Studying Immersive Effects of Game Audio Perception on Player Experiences in Virtual Reality. In Proceedings of the 2018 CHI Conference on Human Factors in Computing Systems (CHI '18). ACM, New York, NY, USA, 328:1-328:13. DOI : http://dx.doi .org/10.1145/3173574.3173902

[144] Robert Rosenthal and Ralph L. Rosnow. 1984. Essentials of Behavioral Research: Methods and Data Analysis. McGraw-Hill, Inc.

[145] D. Roth, C. Klelnbeck, T. Feigl, C. Mutschler, and M. E. Latoschik. 2018. Beyond Replication: Augmenting Social Behaviors in Multi-User Virtual Realities. In 2018 IEEE Conference on Virtual Reality and 3D User Interfaces (VR). 215-222. DOI: http://dx.doi.org/10/gf39p9
[146] Barbara Olasov Rothbaum, Larry Hodges, Samantha Smith, Jeong Hwan Lee, and Larry Price. 2000. A Controlled Study of Virtual Reality Exposure Therapy for the Fear of Flying. Journal of Consulting and Clinical Psychology 68, 6 (2000), 1020-1026. DOI : http://dx.doi.org/10/dshf4v

[147] Roy A. Ruddle, Stephen J. Payne, and Dylan M. Jones. 1999. Navigating Large-Scale Virtual Environments: What Differences Occur Between Helmet-Mounted and Desk-Top Displays? Presence: Teleoperators and Virtual Environments 8, 2 (April 1999), 157-168. D0I: http://dx.doi.org/10/dg4s2q

[148] Majed Samad, Elia Gatti, Anne Hermes, Hrvoje Benko, and Cesare Parise. 2019. Pseudo-Haptic Weight: Changing the Perceived Weight of Virtual Objects By Manipulating Control-Display Ratio. In Proceedings of the 2019 CHI Conference on Human Factors in Computing Systems (CHI '19). ACM, Glasgow, Scotland Uk, 320:1-320:13. DOI : http://dx.doi.org/10/gf2hjv

[149] James B. Sampson. 1993. Cognitive Performance of Individuals Using a Head-Mounted Display While Walking. Proceedings of the Human Factors and Ergonomics Society Annual Meeting 37, 4 (Oct. 1993), 338-342. DOI : http://dx.doi .org/10/gd8cg6

[150] Maria V. Sanchez-Vives and Mel Slater. 2005. From Presence to Consciousness through Virtual Reality. Nature Reviews Neuroscience 6, 4 (April 2005), 332. DOI : http://dx. doi .org/10/bh8pn6

[151] Shyam Prathish Sargunam, Kasra Rahimi Moghadam, Mohamed Suhail, and Eric D. Ragan. 2017. Guided Head Rotation and Amplified Head Rotation: Evaluating Semi-Natural Travel and Viewing Techniques in Virtual Reality. IEEE, 19-28. DOI : http://dx.doi.org/10.1109/VR.2017.7892227

[152] Willem E. Saris and Irmtraud N. Gallhofer. 2014. Design, Evaluation, and Analysis of Questionnaires for Survey Research. John Wiley \& Sons.

[153] Samuel B. Schorr and Allison M. Okamura. 2017. Fingertip Tactile Devices for Virtual Object Manipulation and Exploration. ACM Press, 3115-3119. DOI : http://dx.doi.org/10.1145/3025453.3025744

[154] Thomas Schubert, Frank Friedmann, and Holger Regenbrecht. 1999. Embodied Presence in Virtual Environments. In Visual Representations and Interpretations, Ray Paton and Irene Neilson (Eds.). Springer London, 269-278.

[155] Thomas Schubert, Frank Friedmann, and Holger Regenbrecht. 2001. The Experience of Presence: Factor Analytic Insights. Presence: Teleoper. Virtual Environ. 10, 3 (June 2001), 266-281. DOI: http://dx.doi.org/10/cvt2nz

[156] Peter Schulz, Dmitry Alexandrovsky, Felix Putze, Rainer Malaka, and Johannes Schöning. 2019. The 
Role of Physical Props in VR Climbing Environments. In Proceedings of the 2019 CHI Conference on Human Factors in Computing Systems (CHI'19). ACM, Glasgow, Scotland Uk, 183:1-183:13. DOI : http://dx.doi.org/10/gf3kx3

[157] Valentin Schwind. 2018. Implications of the Uncanny Valley of Avatars and Virtual Characters for Human-Computer Interaction. (2018). DOI : http://dx . doi.org/10/gd6b7h

[158] Valentin Schwind, Pascal Knierim, Lewis Chuang, and Niels Henze. 2017. "Where's Pinky?": The Effects of a Reduced Number of Fingers in Virtual Reality. ACM Press, 507-515. DOI :

http://dx.doi.org/10.1145/3116595.3116596

[159] Valentin Schwind, Pascal Knierim, Nico Haas, and Niels Henze. 2019. Using Presence Questionnaires in Virtual Reality. In Proceedings of the 2019 CHI Conference on Human Factors in Computing Systems (CHI '19). ACM, Glasgow, Scotland Uk, 360:1-360:12. DOI : http://dx. doi .org/10/gfz8sr

[160] Valentin Schwind, Pascal Knierim, Cagri Tasci, Patrick Franczak, Nico Haas, and Niels Henze. 2017. "These Are Not My Hands!": Effect of Gender on the Perception of Avatar Hands in Virtual Reality. ACM Press, 1577-1582. DOI : http://dx.doi.org/10.1145/3025453.3025602

[161] Valentin Schwind, Sven Mayer, Alexandre Comeau-Vermeersch, Robin Schweigert, and Niels Henze. 2018. Up to the Finger Tip: The Effect of Avatars on Mid-Air Pointing Accuracy in Virtual Reality. In Proceedings of the 2018 Annual Symposium on Computer-Human Interaction in Play (CHI PLAY '18). ACM, Melbourne, VIC, Australia, 477-488. DOI : http://dx.doi.org/10/gf3ngj

[162] Sven Seele, Sebastian Misztal, Helmut Buhler, Rainer Herpers, and Jonas Schild. 2017. Here's Looking At You Anyway!: How Important Is Realistic Gaze Behavior in Co-Located Social Virtual Reality Games? ACM Press, 531-540. DOI : http://dx.doi.org/10.1145/3116595.3116619

[163] John J. Shaughnessy and Eugene B. Zechmeister. 1985. Research Methods in Psychology. Alfred A. Knopf, New York, NY, US.

[164] Emily Shaw, Tessa Roper, Tommy Nilsson, Glyn Lawson, Sue V.G. Cobb, and Daniel Miller. 2019. The Heat Is On: Exploring User Behaviour in a Multisensory Virtual Environment for Fire Evacuation. In Proceedings of the 2019 CHI Conference on Human Factors in Computing Systems (CHI '19). ACM, Glasgow, Scotland Uk, 626:1-626:13. DOI : http://dx.doi.org/10/gf3kx9

[165] Lindsay Alexander Shaw, Burkhard Claus WÃCEnsche, Christof Lutteroth, S. Marks, and Rodolphe Callies. 2015. Challenges in Virtual Reality Exergame Design. 162 (Jan. 2015).
[166] Thomas B. Sheridan. 1992. Musings on Telepresence and Virtual Presence. Presence: Teleoperators and Virtual Environments 1, 1 (Jan. 1992), 120-126. DOI : http://dx.doi.org/10/gdcftg

[167] Jotaro Shigeyama, Takeru Hashimoto, Shigeo Yoshida, Takuji Narumi, Tomohiro Tanikawa, and Michitaka Hirose. 2019. Transcalibur: A Weight Shifting Virtual Reality Controller for 2D Shape Rendering Based on Computational Perception Model. In Proceedings of the 2019 CHI Conference on Human Factors in Computing Systems (CHI '19). ACM, Glasgow, Scotland Uk, 11:1-11:11. DOI :

http://dx.doi.org/10/gfz8qr

[168] Ben Shneiderman, Catherine Plaisant, Maxine Cohen, Steven Jacobs, Niklas Elmqvist, and Nicholas Diakopoulos. 2016. Designing the User Interface: Strategies for Effective Human-Computer Interaction (6 edition ed.). Pearson, Boston.

[169] Valerie J. Shute. 2011. Stealth Assessment in Computer-Based Games to Support Learning. Computer games and instruction 55, 2 (2011), 503-524.

[170] Mel Slater. 2009. Place Illusion and Plausibility Can Lead to Realistic Behaviour in Immersive Virtual Environments. Philosophical Transactions of the Royal Society B: Biological Sciences 364, 1535 (Dec. 2009), 3549-3557. DOI : http://dx.doi.org/10/df44xc

[171] Mel Slater and Anthony Steed. 2000. A Virtual Presence Counter. Presence: Teleoperators and Virtual Environments 9, 5 (2000), 413-434. DOI : http://dx.doi.org/10/cd5kxg

[172] Mel Slater, Martin Usoh, and Yiorgos Chrysanthou. 1995. The Influence of Dynamic Shadows on Presence in Immersive Virtual Environments. In Virtual Environments '95 (Eurographics), Martin Göbel (Ed.). Springer Vienna, 8-21.

[173] Jeongmin Son, Sunggeun Ahn, Sunbum Kim, and Geehyuk Lee. 2019. Improving Two-Thumb Touchpad Typing in Virtual Reality. In Extended Abstracts of the 2019 CHI Conference on Human Factors in Computing Systems (CHI EA '19). ACM, Glasgow, Scotland Uk, LBW2217:1-LBW2217:6. DOI : http://dx.doi.org/10/gf3kb9

[174] Maurício Sousa, Daniel Mendes, Soraia Paulo, Nuno Matela, Joaquim Jorge, and Daniel Simões Lopes. 2017. VRRRRoom: Virtual Reality for Radiologists in the Reading Room. ACM Press, 4057-4062. DOI: http://dx.doi.org/10.1145/3025453.3025566

[175] Beatriz Sousa Santos, Paulo Dias, Angela Pimentel, Jan-Willem Baggerman, Carlos Ferreira, Samuel Silva, and Joaquim Madeira. 2008. Head-Mounted Display versus Desktop for 3D Navigation in Virtual Reality: A User Study. Multimedia Tools and Applications 41, 1 (Aug. 2008), 161. DOI : http://dx.doi.org/10/ccq4dq 
[176] Marco Speicher, Jan Ehrlich, Vito Gentile, Donald Degraen, Salvatore Sorce, and Antonio Krüger. 2019. Pseudo-Haptic Controls for Mid-Air Finger-Based Menu Interaction. In Extended Abstracts of the 2019 CHI Conference on Human Factors in Computing Systems (CHI EA '19). ACM, Glasgow, Scotland Uk, LBW0285:1-LBW0285:6. DOI: http://dx.doi.org/10/gf3kcj

[177] Marco Speicher, Anna Maria Feit, Pascal Ziegler, and Antonio Krüger. 2018. Selection-Based Text Entry in Virtual Reality. In Proceedings of the 2018 CHI Conference on Human Factors in Computing Systems (CHI '18). ACM, New York, NY, USA, 647:1-647:13. DOI: http://dx.doi .org/10.1145/3173574.3174221

[178] Misha Sra, Xuhai Xu, and Pattie Maes. 2018. BreathVR: Leveraging Breathing As a Directly Controlled Interface for Virtual Reality Games. In Proceedings of the 2018 CHI Conference on Human Factors in Computing Systems (CHI '18). ACM, New York, NY, USA, 340:1-340:12. DOI : http://dx . doi .org/10.1145/3173574.3173914

[179] J. Stauffert, F. Niebling, and M. E. Latoschik. 2018. Effects of Latency Jitter on Simulator Sickness in a Search Task. In 2018 IEEE Conference on Virtual Reality and $3 D$ User Interfaces (VR). 121-127. DOI: http://dx.doi.org/10/gf39qf

[180] Carolin Straßmann, Sabrina C. Eimler, Alexander Arntz, Dustin Keßler, Sarah Zielinski, Gabriel Brandenberg, Vanessa Dümpel, and Uwe Handmann. 2019. Relax Yourself - Using Virtual Reality to Enhance Employees' Mental Health and Work Performance. In Extended Abstracts of the 2019 CHI Conference on Human Factors in Computing Systems (CHI EA '19). ACM, Glasgow, Scotland Uk, LBW0286:1-LBW0286:6. DOI: http://dx.doi .org/10/gf3kch

[181] Hemant Bhaskar Surale, Aakar Gupta, Mark Hancock, and Daniel Vogel. 2019a. TabletInVR: Exploring the Design Space for Using a Multi-Touch Tablet in Virtual Reality. In Proceedings of the 2019 CHI Conference on Human Factors in Computing Systems (CHI '19). ACM, Glasgow, Scotland Uk, 13:1-13:13. DOI: http://dx.doi.org/10/gf2d42

[182] Hemant Bhaskar Surale, Fabrice Matulic, and Daniel Vogel. 2019b. Experimental Analysis of Barehand Mid-Air Mode-Switching Techniques in Virtual Reality. In Proceedings of the 2019 CHI Conference on Human Factors in Computing Systems (CHI '19). ACM, Glasgow, Scotland Uk, 196:1-196:14. DOI : http://dx . doi .org/10/gf3kx2

[183] Penelope Sweetser and Peta Wyeth. 2005. GameFlow: A Model for Evaluating Player Enjoyment in Games. Comput. Entertain. 3, 3 (July 2005), 3-3. DOI: http://dx. doi .org/10/d4k4x4

[184] Theophilus Teo, Louise Lawrence, Gun A. Lee, Mark Billinghurst, and Matt Adcock. 2019. Mixed Reality
Remote Collaboration Combining 360 Video and 3D Reconstruction. In Proceedings of the 2019 CHI Conference on Human Factors in Computing Systems (CHI '19). ACM, Glasgow, Scotland Uk, 201:1-201:14. DOI : http://dx.doi.org/10/gf2bzn

[185] Balasaravanan Thoravi Kumaravel, Cuong Nguyen, Stephen DiVerdi, and Björn Hartmann. 2019.

TutoriVR: A Video-Based Tutorial System for Design Applications in Virtual Reality. In Proceedings of the 2019 CHI Conference on Human Factors in Computing Systems (CHI '19). ACM, Glasgow, Scotland Uk, 284:1-284:12. DOI: http://dx.doi.org/10/gf3krs

[186] Tanh Quang Tran, HyunJu Shin, Wolfgang Stuerzlinger, and JungHyun Han. 2017. Effects of Virtual Arm Representations on Interaction in Virtual Environments. ACM Press, 1-9. DOI : http://dx.doi.org/10.1145/3139131.3139149

[187] Sam Tregillus, Majed Al Zayer, and Eelke Folmer. 2017. Handsfree Omnidirectional VR Navigation Using Head Tilt. ACM Press, 4063-4068. DOI : http://dx.doi.org/10.1145/3025453.3025521

[188] Hsin-Ruey Tsai, Jun Rekimoto, and Bing-Yu Chen. 2019. ElasticVR: Providing Multilevel Continuously-Changing Resistive Force and Instant Impact Using Elasticity for VR. In Proceedings of the 2019 CHI Conference on Human Factors in Computing Systems (CHI '19). ACM, Glasgow, Scotland Uk, 220:1-220:10. DOI : http://dx. doi .org/10/gf2hjr

[189] Martin Usoh, Ernest Catena, Sima Arman, and Mel Slater. 2000. Using Presence Questionnaires in Reality. Presence: Teleoperators and Virtual Environments 9, 5 (2000), 497-503. DOI : http://dx.doi . org/10/bf3cmp

[190] Adrien Verhulst, Jean-Marie Normand, Cindy Lombart, and Guillaume Moreau. 2017. A Study on the Use of an Immersive Virtual Reality Store to Investigate Consumer Perceptions and Purchase Behavior toward Non-Standard Fruits and Vegetables. IEEE, 55-63. DOI : http://dx.doi .org/10.1109/VR. 2017.7892231

[191] Alexandra Voit, Sven Mayer, Valentin Schwind, and Niels Henze. 2019. Online, VR, AR, Lab, and In-Situ: Comparison of Research Methods to Evaluate Smart Artifacts. In Proceedings of the 2019 CHI Conference on Human Factors in Computing Systems (CHI '19). ACM, Glasgow, Scotland Uk, 507:1-507:12. DOI: http://dx.doi.org/10.1145/3290605.3300737

[192] Emanuel Vonach, Clemens Gatterer, and Hannes Kaufmann. 2017. VRRobot: Robot Actuated Props in an Infinite Virtual Environment. IEEE, 74-83. DOI: http://dx.doi.org/10.1109/VR. 2017.7892233

[193] Daniel Wagner, Christian Hofmann, Heiko Hamann, and Sebastian von Mammen. 2017. Design and Exploration of Braiding Swarms in VR. ACM Press, 1-4. DOI: http://dx.doi.org/10.1145/3139131. 3139169

[194] Ryan Wedoff, Lindsay Ball, Amelia Wang, Yi Xuan Khoo, Lauren Lieberman, and Kyle Rector. 2019. 
Virtual Showdown: An Accessible Virtual Reality Game with Scaffolds for Youth with Visual Impairments. In Proceedings of the 2019 CHI Conference on Human Factors in Computing Systems (CHI '19). ACM, Glasgow, Scotland Uk, 141:1-141:15. DOI : http://dx.doi .org/10/gf2nfp

[195] Florian Weidner, Anne Hoesch, Sandra Poeschl, and Wolfgang Broll. 2017. Comparing VR and Non-VR Driving Simulations: An Experimental User Study. IEEE, 281-282. DOI :

http://dx.doi.org/10.1109/VR. 2017.7892286

[196] T. Weißker, A. Kunert, B. Fröhlich, and A. Kulik. 2018. Spatial Updating and Simulator Sickness During Steering and Jumping in Immersive Virtual Environments. In 2018 IEEE Conference on Virtual Reality and 3D User Interfaces (VR). 97-104. DOI: http://dx.doi.org/10/gf39qj

[197] Martin Westhoven, Dennis Paul, and Thomas Alexander. 2016. Head Turn Scaling below the Threshold of Perception in Immersive Virtual Environments. ACM Press, 77-86. DOI : http://dx.doi .org/10.1145/2993369.2993385

[198] C. Wienrich, K. Schindler, N. Döllinqer, S. Kock, and O. Traupe. 2018. Social Presence and Cooperation in Large-Scale Multi-User Virtual Reality - The Relevance of Social Interdependence for Location-Based Environments. In 2018 IEEE Conference on Virtual Reality and 3D User Interfaces (VR). 207-214. DOI : http://dx.doi .org/10/gf39qb

[199] Graham Wilson and Mark McGill. 2018. Violent Video Games in Virtual Reality: Re-Evaluating the Impact and Rating of Interactive Experiences. In Proceedings of the 2018 Annual Symposium on Computer-Human Interaction in Play (CHI PLAY' '18). ACM, Melbourne, VIC, Australia, 535-548. DOI :

http://dx.doi.org/10/gf3ngh
[200] Graham Wilson, Mark McGill, Matthew Jamieson, Julie R. Williamson, and Stephen A. Brewster. 2018. Object Manipulation in Virtual Reality Under Increasing Levels of Translational Gain. In Proceedings of the 2018 CHI Conference on Human Factors in Computing Systems (CHI '18). ACM, New York, NY, USA, 99:1-99:13. DOI :

http://dx.doi .org/10.1145/3173574.3173673

[201] Bob G. Witmer and Michael J. Singer. 1998. Measuring Presence in Virtual Environments: A Presence Questionnaire. Presence: Teleoperators and Virtual Environments 7, 3 (June 1998), 225-240. DOI: http://dx.doi.org/10/dw9b5s

[202] Soojeong Yoo and Judy Kay. 2016. VRun: Running-in-Place Virtual Reality Exergame. In Proceedings of the 28th Australian Conference on Computer-Human Interaction (OzCHI '16). ACM, New York, NY, USA, 562-566. DOI: http://dx.doi.org/10.1145/3010915.3010987

[203] Majed Al Zayer. 2019. Universal Usability of Virtual Reality. Dissertation. University of Nevada, Reno, Nevada, Reno.

[204] André Zenner and Antonio Krüger. 2019. Drag:On: A Virtual Reality Controller Providing Haptic Feedback Based on Drag and Weight Shift. In Proceedings of the 2019 CHI Conference on Human Factors in Computing Systems (CHI '19). ACM, Glasgow, Scotland Uk, 211:1-211:12. DOI : http://dx.doi.org/10/gf2hjs

[205] Yuhang Zhao, Edward Cutrell, Christian Holz, Meredith Ringel Morris, Eyal Ofek, and Andrew D. Wilson. 2019. SeeingVR: A Set of Tools to Make Virtual Reality More Accessible to People with Low Vision. In Proceedings of the 2019 CHI Conference on Human Factors in Computing Systems (CHI '19). ACM, Glasgow, Scotland Uk, 111:1-111:14. DOI : http://dx.doi.org/10/gf2nfg 